Antonio Rafael

Peña Sánchez*

Mercedes

Jiménez García **

José

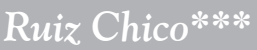

Universidad de Cádiz, Cádiz, España

Recibido: 12 de octubre de 2013

Concepto de evaluación: 22 de enero de 2014

Aprobado: 17 de marzo de 2014

Artículo de investigación DOI: $h t t p: / / d x$.doi.org/10.14718/ revfinanzpolitecon.2014.6.1.3 (c) 2014 Universidad Católica de Colombia. Facultad de Ciencias Económicas y Administrativas. Todos los derechos reservados.

*Doctor en Economía. Profesor titular de la Universidad de Cádiz en el Área de Economía Aplicada.

Vicedecano de Comunicación y

Relaciones Institucionales, Coordinador del Máster Universitario en Gestión y Administración Pública, Facultad de Ciencias Sociales y de la Comunicación. Dirección de correspondencia: Universidad

de Cádiz, Campus de Jerez. Avda. de la Universidad, s/n, 11405 Jerez de la Frontera, Cádiz, España. Correo electrónico: rafael.pena@uca.es

**Doctora en Economía. Profesora ayudante. Doctora de la Universidad de Cádiz en el Área de Economía Aplicada. Facultad de Ciencias Económicas y Empresariales. Dirección de correspondencia: Universidad de Cádiz, Campus de Cádiz, Glorieta Carlos Cano, s/n, 11002

Cádiz, España. Correo electrónico: mercedes.jimenezgarcia@uca.es

***Doctor en Economía. Profesor Sustituto de la Universidad de Cádiz en el Área de Economía Aplicada, de la Facultad de Ciencias Sociales y de la Comunicación. Dirección de correspondencia: Universidad de Cádiz, Campus de Jerez. Avda. de la Universidad, s/n, 11405 Jerez de la Frontera, Cádiz, España. Correo electrónico: jose.ruizchico@uca.es
Finanz. polit. econ., ISSN: 2248-6046, Vol. 6, No. 1, enero-junio, 2014, pp. 43-71

Edición Especial - Economía Regional

\section{Impacto de la crisis económica en el empleo de las regiones españolas: un análisis sectorial en el periodo 2007-2010}

\section{RESUMEN}

El objetivo de este trabajo es analizar el impacto que la crisis económica ha tenido en las distintas regiones y en los sectores de la economía española durante el periodo 2007-2010, medido a través del empleo y el capital humano. Para tal fin, se ha estudiado la evolución del mercado de trabajo y el capital humano en los últimos años. El destacado descenso experimentado por el empleo, así como en menor medida por el capital humano, pone de manifiesto la vulnerabilidad del tejido productivo y empresarial de las regiones españolas. No obstante, los resultados que arrojan el estudio planteado sostienen que la crisis económica ha afectado de forma desigual a las regiones españolas; esta se ha intensificado, principalmente, en aquellos territorios con mayor especialización en sectores como la construcción, la industria y los servicios destinados a la venta.

Palabras clave: crisis económica, análisis regional, estudio sectorial, Unión Económica y Monetaria.

JEL: D24, J24, O18, R11

\section{Impact of the Economic Crisis on Employment in Spanish Regions: Analysis by Sector for the Years 2007-2010}

\section{ABSTRACT}

The aim of this study is to analyze the impact that the global economic crisis has had in the various regions and sectors of the Spanish Economy during the years 2007-2010, measured in terms of jobs and human capital. To this end the evolution of the labour market and human capital in recent years is examined. The increase in unemployment, and to a lesser extent, human capital, demonstrates the vulnerability of business and production in the regions of Spain. Nevertheless, the results of the study show that the crisis has had an irregular effect across regions, being greater in those regions that depend more on industries such as construction and industry and service relating to retail.

Keywords: Economic crisis, regional analysis, sector study, Economic and Monetary Union. 


\section{Impacto da crise econômica no emprego das regiões espanholas: uma análise setorial no período 2007-2010}

\section{RESUMO}

O objetivo deste trabalho é analisar o impacto que a crise econômica teve nas diferentes regiões e nos setores da economia espanhola durante o período 2007-2010, medido por meio do emprego e do capital humano. Para tal fim, tem-se estudado a evolução do mercado de trabalho e do capital humano nos últimos anos. A destacada queda experimentada pelo emprego e, em menor medida, pelo capital humano, manifesta a vulnerabilidade do tecido produtivo e empresarial das regiões espanholas. Contudo, os resultados que produzem o estudo apresentado sustentam que a crise econômica afetou de forma desigual as regiões espanholas; esta se intensificou, principalmente, naqueles territórios com maior especialização em setores como a construção, a indústria e os serviços destinados à venda.

Palavras-chave: crise econômica, análise regional, estudo setorial, União Econômica e Monetária. 


\section{INTRODUCCIÓN}

Si existe algún fenómeno que a lo largo del siglo XXI haya cubierto más páginas en la literatura económica ha sido, sin duda, la crisis económica que vienen padeciendo los países en el mundo. Este ha sido un aspecto ampliamente estudiado y debatido por especialistas del campo, no solo de la economía, sino también de otras disciplinas como la política, la sociología, la comunicación, etc. Aunque es muy abundante la cantidad de textos, documentos y trabajos que se han publicado sobre las causas de la crisis y sus consecuencias en el nivel de vida y de bienestar de los ciudadanos a nivel mundial, aún quedan aspectos interesantes sobre los que todavía no se ha indagado con profundidad.

A estas alturas del proceso, nadie pone en cuestión que el origen de la crisis comenzó en 2007, y desde entonces dicha circunstancia se viene agravando sistemáticamente. Aunque la situación no ha sido igual en todos los países, sí es cierto que en la Unión Económica y Monetaria (UEM) algunos países se han visto más intensamente afectados, como es el caso de Grecia, Portugal, Irlanda, España e Italia.

En el caso de España, la crisis económica ha puesto al descubierto la vulnerabilidad de su tejido productivo y empresarial, y esto se ha manifestado a través de la elevada tasa de desempleo que viene padeciendo en los últimos años, y que en 2012 ha llegado a alcanzar una cifra cercana al $25 \%$. Esta crisis no ha afectado de la misma manera a todas las regiones españolas (Fernández y Morán, 2008; Ruiz-Huerta et al., 2012)2 ${ }^{2}$ lo que ha puesto en peligro el proceso de cohesión territorial que se ha intentado conseguir en los últimos 30 años.

$1 \quad$ Los autores del trabajo agradecen las sugerencias y comentarios recibidos por los evaluadores de la revista Finanzas y Política Económica, los cuales, sin duda, han permitido mejorar sustancialmente el contenido de la presente investigación. No obstante, cualquier error es responsabilidad exclusiva de los autores.

2 Este aspecto fue intensamente debatido en las múltiples comunicaciones presentadas en las V Jornadas de Geografía Económica de Girona, celebradas del 28 al 30 de junio de 2012. Véase al respecto la publicación de dichos trabajos en Llussá et al. (2013).
Tampoco ha sido similar en todos los sectores económicos (Cabrales et al., 2009; Xiaoni et al., 2011; Laparra y Pérez, 2012), por lo que se verán más perjudicadas aquellas regiones a las que les haya afectado más en sus sectores energético e industrial, pues son los que mayor productividad aparente del empleo aportan, y en el sector construcción, por ser un sector que utiliza de forma abundante e intensivamente el factor trabajo.

Teniendo en cuenta las premisas expuestas anteriormente, el objetivo que se pretende abordar en este trabajo es analizar el impacto que la crisis económica ha tenido en las distintas regiones y en los distintos sectores productivos de la economía española durante el periodo 2007-20103, medido a través del empleo y del capital humano, con el fin de establecer una tipología de regiones en función de la repercusión que sobre éstas haya tenido la crisis económica y financiera. En este sentido, se trata de contrastar la hipótesis de que las regiones españolas han sufrido las consecuencias de la crisis económica de forma diferente, siendo el impacto distinto en función de la especialización productiva de cada una de ellas.

El aporte realizado por este trabajo consiste en poner de manifiesto la debilidad de la actividad económica de algunas regiones españolas, distinguiéndose del resto de las regiones por la intensidad con que ha azotado e impactado la crisis económica-financiera en su estructura productiva, valorada esta última a partir de su estructura sectorial del empleo y del capital humano. Lo anterior permite aportar nuevos datos y nuevos elementos de juicio sobre uno de los problemas importantes de la economía española, como son las diferencias $\tan$ acusadas existentes entre las distintas regiones (Peña, 2007, 2008a, 2008b, 2011; Peña y Jiménez, 2012, 2013)

Para el fin propuesto en esta investigación, el trabajo se estructura de la siguiente forma. En el segundo apartado se enumeran las fuentes

3 El periodo considerado ha sido el 2007-2010 debido a la homogeneidad que presentan los datos analizados, teniendo en cuenta las fuentes estadísticas utilizadas, aspecto que se señala en el segundo apartado de este documento. 
estadísticas utilizadas en el presente estudio. En el tercer apartado se valora el impacto que la crisis económico-financiera mundial ha tenido sobre la economía española, contextualizándola tanto en el tiempo como en su situación dentro de la Unión Europea. En el cuarto apartado se realiza un análisis de la repercusión que la crisis ha tenido en las regiones españolas. En el quinto apartado se estudia el efecto de la crisis en la estructura sectorial de las regiones españolas. $Y$ en la parte final se reúnen las principales ideas y conclusiones de la investigación efectuada.

\section{FUENTES ESTADÍSTICAS CONSULTADAS}

Las fuentes estadísticas consultadas para la elaboración de la presente investigación han sido las siguientes: a) Contabilidad Regional del Instituto Nacional de Estadística (INE) (http://www.ine.es); b) bases de datos de la Secretaría de Estado de Presupuestos y Gastos del Ministerio de Hacienda y Administraciones Públicas del Gobierno de España (http://www.sepg.pap.meh.es): BD.MORES, base de datos regional, actualizada en diciembre de 2011 (Dabán et al., 2002); BDREMS, base de datos trimestral, actualizada en diciembre de 2012; BDMACRO, base de datos anual, actualizada en mayo de 2011 (Díaz y García, 2011); c) estadísticas de Eurostat (http://epp.eurostat.ec.europa.eu-/ portal/page/portal/statistics/themes); d) Base de datos de Capital Humano del Instituto Valenciano de Investigaciones Económicas (IVIE) (http:// www.ivie.es); y e) El Balance Económico Regional (Autonomías y Provincias). Años 2000 a 2010, de la Fundación de Cajas de Ahorros (Alcaide, 2011). Con el fin de evitar distorsiones, se han eliminado del análisis las ciudades autónomas de Ceuta y Melilla.

La utilización de las distintas bases de datos anteriormente referenciadas permite abordar un análisis riguroso y científico, con datos homogéneos, que puede arrojar luz sobre el objetivo propuesto en este trabajo, e incluso corroborar conclusiones de otros documentos publicados en los que se han utilizado distintas fuentes estadísticas.
Además, como se ha puesto de manifiesto anteriormente, para llevar a cabo este estudio se ha recurrido al uso de diversas fuentes de información que se señalan claramente en las correspondientes tablas y gráficos de este trabajo. Por lo anterior, es necesario tener en cuenta que la utilización de fuentes estadísticas heterogéneas puede hacer que algunas cifras concretas que aparecen en la investigación difieran ligeramente, y se estima que las mismas no son particularmente relevantes.

\section{EL DESCENSO DE LA ACTIVIDAD ECONÓMICA DE ESPAÑA EN EL CONTEXTO DE LA UNIÓN EUROPEA}

En este apartado se desarrollarán un par de cuestiones importantes para contextualizar la investigación que se presenta. En primer lugar, se intenta fijar el periodo temporal de análisis. Para ello se determina, mediante la observación de la evolución temporal de ciertas variables económicas, como son el Producto Interior Bruto (PIB a partir de ahora) y su derivada por habitante (PIBpc), el empleo generado en la economía, la tasa de desempleo (TD) y el número de horas empleadas en la actividad productiva, cuando comienza la desaceleración económica en España, de forma que se pueda acotar con claridad el periodo que se va a examinar. En segundo lugar, se trata de determinar la repercusión de la crisis económicofinanciera en los países de la Unión Europea, con el fin de encuadrar la situación en la que se encuentra España en el contexto europeo.

La crisis económica se pone de manifiesto en España a partir de 2008, con una fuerte caída de la actividad económica, como se puede apreciar en la gráfica 1. El PIB, valorado a precios constantes de 2000, experimenta un intenso retroceso de 3,7 puntos porcentuales en 2009 y un 0,1 punto porcentual en 2010. Tras el amplio periodo de auge económico en el periodo 1994-2008, con un crecimiento anual acumulativo de 2,9 puntos porcentuales, la caída experimentada a partir de 2008 representa un intenso deterioro de la actividad productiva en la economía española. 
Lógicamente, el proceso anterior ha supuesto un destacado descenso de la ocupación del 6,6\% en 2009 y del 2,3\% en 2010 (gráfica 2). Este hecho se hace más apreciable cuando se observa que desde 1994 y hasta 2007 la misma variable había experimentado un crecimiento sostenido de un $3,4 \%$ anual acumulativo. Por tanto, se aprecia una intensa destrucción de empleos en la economía española en los dos últimos años estudiados.

Gráfica 1.

Evolución del PIB

(precios constantes de 2000)

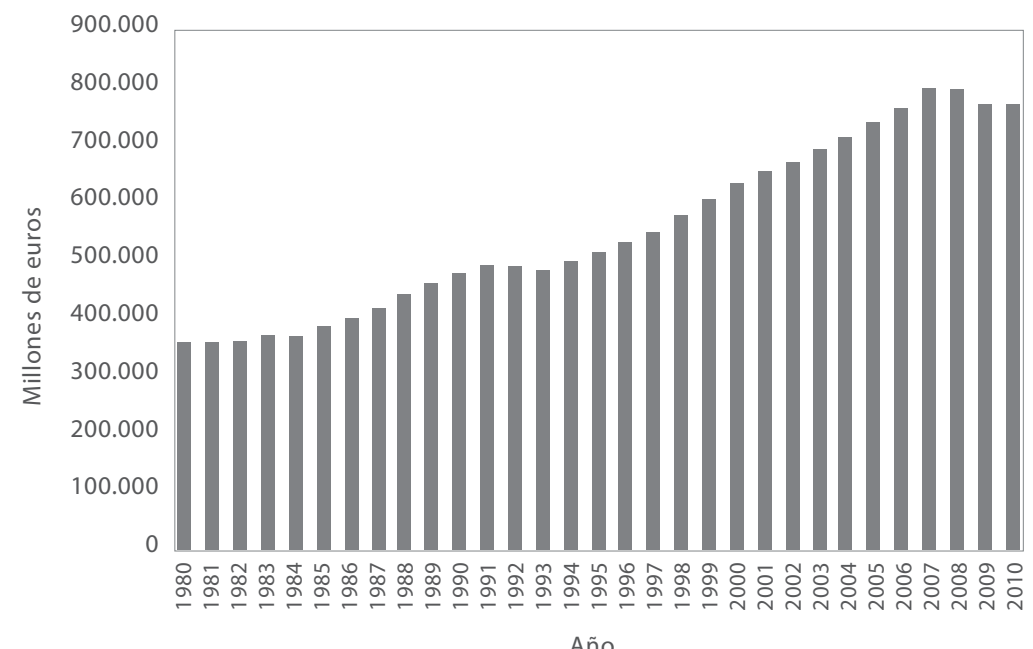

Año

Fuente: elaboración de los autores, con base en datos de Bdmacro.

Gráfica 2.

\section{Evolución del empleo}

(miles de empleos)

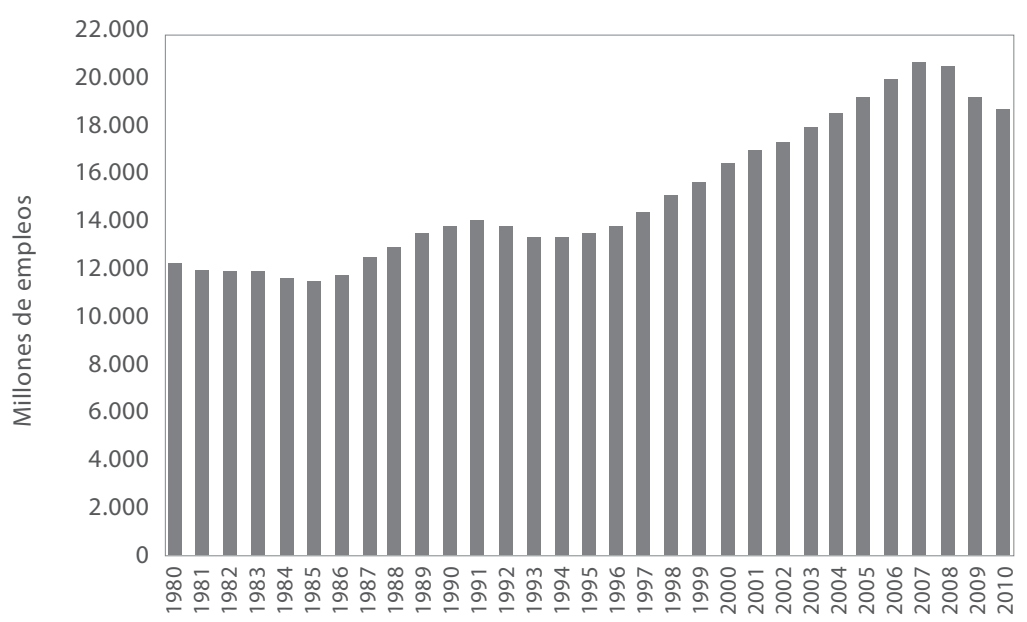

Año

Fuente: elaboración de los autores, con base en datos de Bdmacro. 
En este sentido, el crecimiento experimentado por la productividad aparente del empleo a partir de 2008 (gráfica 3) ha venido motivado, fundamentalmente, por la intensa reducción experimentada por el empleo, y no como resultado de la acumulación de ganancias en eficiencia productiva; este último es un aspecto crucial en el logro de aumentos sostenidos de competitividad (Cuadrado y Maroto, 2012). En definitiva, el lento incremento de la productividad del empleo viene explicado fundamentalmente por el abarata- miento del despido debido a la reforma del mercado laboral, al uso más frecuente de la contratación temporal y a la entrada de inmigrantes ${ }^{4}$, y por consiguiente descansa en los cambios que se han producido en el mercado de trabajo. Este aumento de la productividad en el periodo 2008-2010 no ha sido lo suficientemente intenso como para permitir un crecimiento o, al menos, el mantenimiento del nivel de desarrollo económico, medido a partir del PIBpc, que como puede observarse ha sufrido una considerable reducción en el periodo considerado.

Gráfica 3.

Evolución del PIB por habitantes y de la productividad aparente del empleo

(Euros constantes del año 2000)

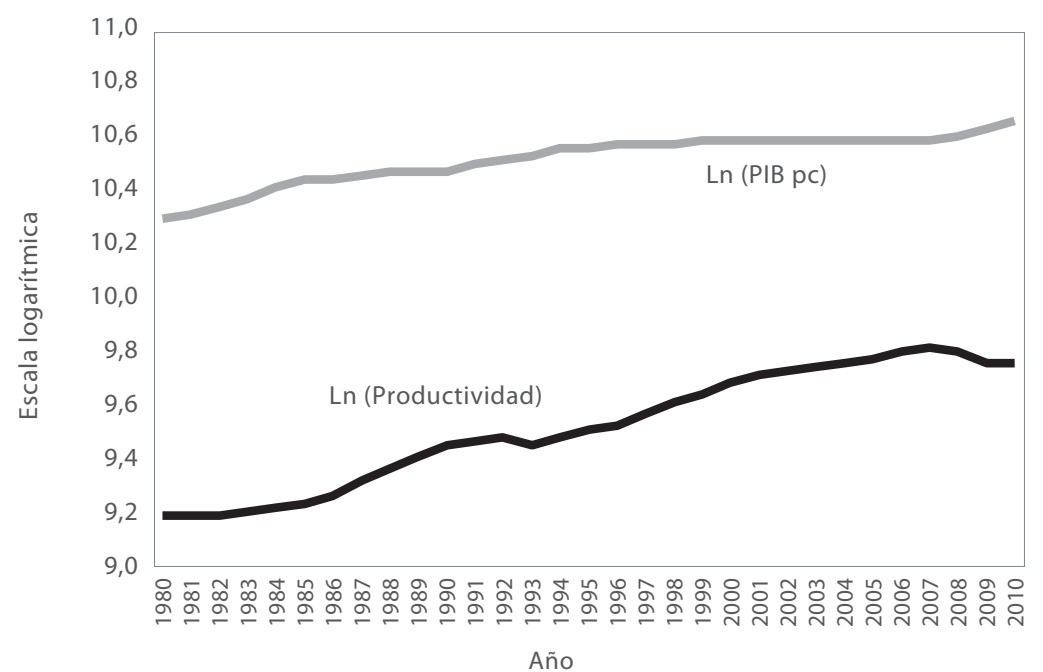

Fuente: elaboración de los autores, con base en datos de Bdmacro.

La expulsión de mano de obra del mercado laboral de 1,9 millones de trabajadores como consecuencia de la disminución de la demanda nacional y la inversión productiva provocó que la tasa de paro ascendiera del 8,3\% en 2007 al 20,1\% en 2010 (gráfica 4), y se ubicó en niveles que no se conocían desde 1994.

4 Con la consiguiente influencia que este último fenómeno tiene en los costes salariales y en las variables laborales (Medina et al., 2010). En esta línea, la teoría económica convencional sostiene que la entrada de inmigrantes favorece la reducción de salarios al disponer el mercado laboral de una mayor cantidad de población activa, indicativa de la oferta de trabajo. 
Evolución de la tasa de paro

(en porcentaje)

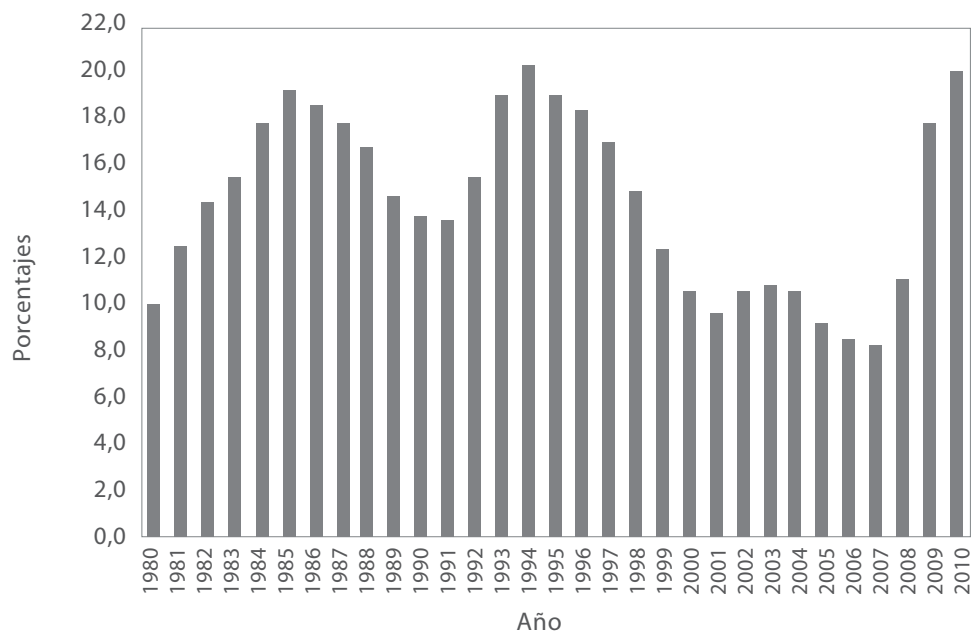

Fuente: elaboración de los autores, con base en datos de Bdmacro.

Gráfica 5.

Evolución de las horas trabajadas

(miles de horas)

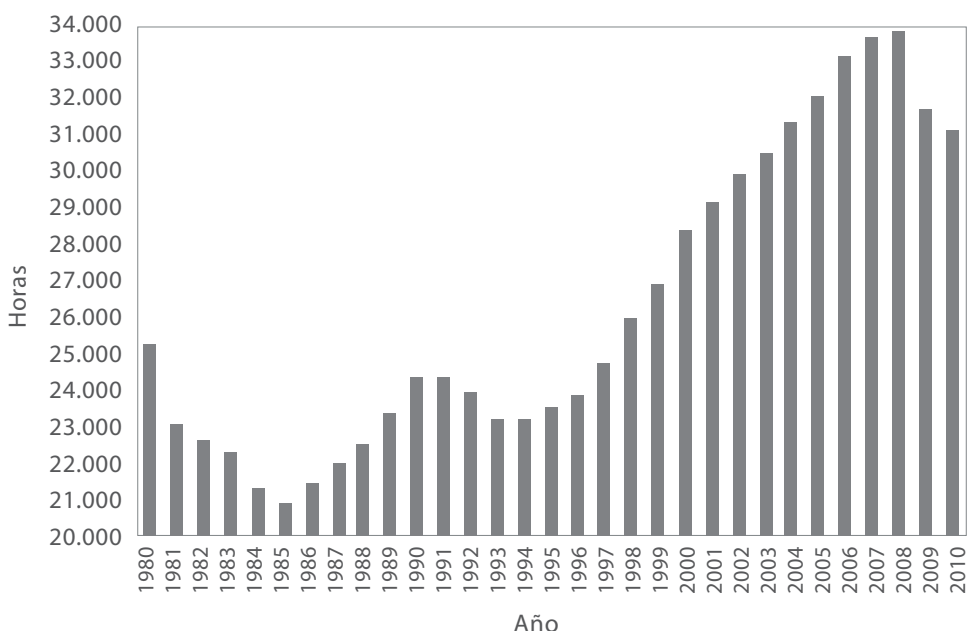

Año

Fuente: elaboración de los autores, con base en datos de Bdmacro.

Si se analiza la fuerte caída de actividad productiva en España en términos de horas trabajadas (gráfica 5) ${ }^{5}$, se observa cómo desde 1994 hasta 2008 se produce un intenso aumento continuado y permanente de las horas trabajadas a una tasa media anual acumulativa de un $2,8 \%$, favorecido

$5 \quad$ Las cuales se relacionan directamente con el número de empleos utilizado en la actividad productiva. en una gran parte por el intenso crecimiento experimentado por el sector de la construcción en este periodo, que actuó como motor y principal demandante de mano de obra exterior (Myro, 2011). En efecto, el crecimiento del sector de la construcción en la economía española favoreció la expansión del PIB, pero aún más la del empleo, al tratarse de un sector intensivo en mano de obra y, por tanto, 
Evolución del PIB trimestral

(precios constantes del año 2000)

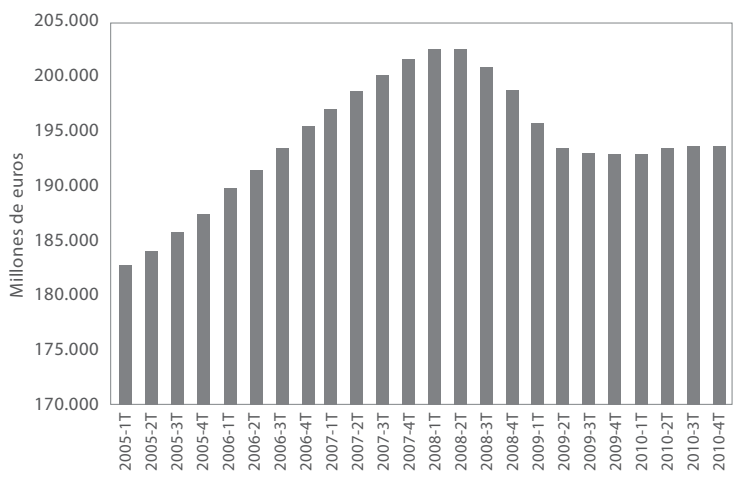

Fuente: elaboración de los autores, con base en datos de Bdrems.

Gráfica 7.

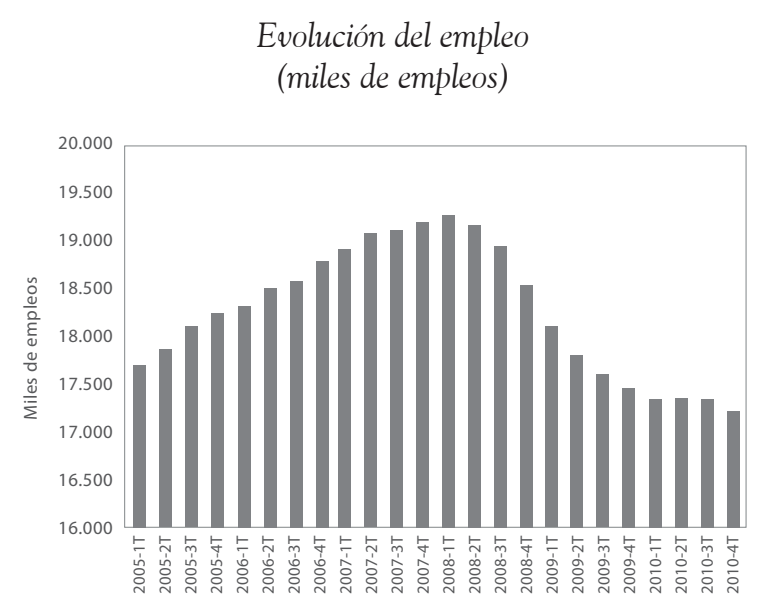

Fuente: elaboración de los autores, con base en datos de Bdrems.

Gráfica 8.

Evolución de las horas trabajadas (miles de horas)

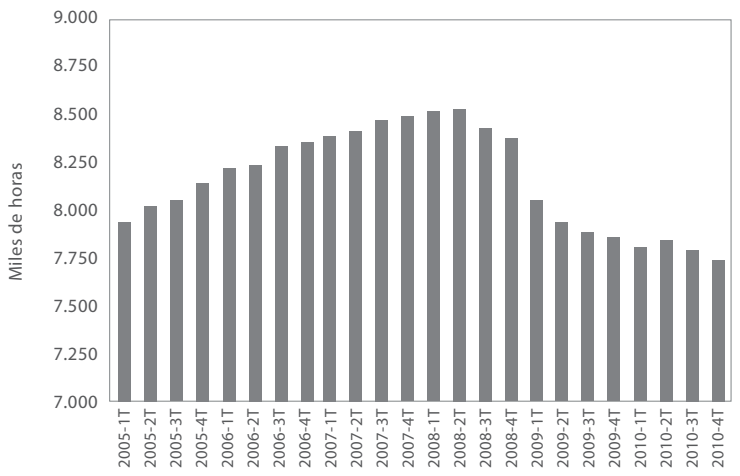

Fuente: elaboración de los autores, con base en datos de Bdrems. 
de baja productividad (Boldrín et al., 2010). Pero a partir de 2008 se produce una intensa caída en la utilización de horas trabajadas, más significativa en 2009 que en 2010. Este descenso se cifra en un 4,01\% anual acumulativo en el periodo 2008-2010, y tras esto alcanzó niveles que no se conocían desde 2004, como ocurría con los niveles de empleo.

Con el fin de fijar con mayor grado de precisión el periodo en el que comienzan a sentirse los efectos de la crisis económica en España, se presentan las gráficas 6, 7 y 8, en las que se recoge la evolución del PIB a precios constantes, del empleo y del número de horas trabajadas en el periodo 2005-2010, por trimestres. Se observa cómo existe una coincidencia generalizada donde la actividad económica comienza a deteriorarse a partir del segundo trimestre de 2008, hecho que coincide con la desaceleración de la demanda de vivienda por parte de los hogares, en respuesta al endurecimiento de las condiciones de financiación y del deterioro del mercado. La reducción de la producción y del empleo en el conjunto de la economía, y la caída de los precios inmobiliarios tuvieron un impacto directo contractivo sobre la renta disponible y la riqueza, lo que desencadenó una serie de efectos de segunda vuelta sobre la inversión residencial, la actividad del sector y de sus industrias complementarias, y nuevamente sobre el empleo (Ortega y Peñalosa, 2012).

Por todo lo anterior, se cree oportuno centrar el estudio en el periodo 2007-2010, ya que al comenzar la desaceleración económica en el segundo trimestre de 2008 ya comienza a deteriorarse el tejido productivo y la actividad de los agentes económicos, por lo que es interesante la comparación entre el año en el que aún no se había producido ningún retroceso (2007) con el último con el se cuenta con datos homogéneos disponibles para estudiar el impacto que la crisis económicofinanciera ha tenido sobre la economía española.

Con la finalidad de contextualizar el proceso de descenso de la actividad económica experimentado por la economía española en el conjunto de la Unión Europea, se presentan los datos de la tabla 1. Esta refleja la enorme debilidad eco- nómica y productiva de España, poniéndose de manifiesto con mayor intensidad en el periodo de crisis económico-financiera que se viene padeciendo en la actualidad. En este sentido, se observa cómo el PIBpc de España ha estado ligeramente por debajo del que presenta la media de la Unión Europa de los 27 miembros (UE-27), aunque la disminución experimentada por España ha sido prácticamente el doble de la observada en la UE27 en el periodo 2007-2010. Además, la tasa de desempleo ha evolucionado con mayor fuerza en España en el mismo periodo, pues si en 2007 la tasa española era un punto superior a la de la UE-27, siendo esta de 7,2 \%, en 2010 consiguió duplicarla, cuando la de la UE-27 era de 9,7\%. Esta volatilidad del empleo no es un fenómeno nuevo en la economía española, pues de hecho el Fondo Monetario Internacional (2010) estimó la elasticidad del desempleo a las variaciones en el PIB para un conjunto amplio de países. Tras esto se encontró que España presentaba una mayor elasticidad entre los países desarrollados (Boura, 2013). Por tanto, este hecho pone de relieve la fuerte debilidad y vulnerabilidad de la economía española frente a los desajustes que se vienen produciendo en el periodo analizado.

Por otro lado, el PIBpc de la UE-27 ha caído cerca de un $1 \%$ anual acumulativo en el periodo 2007-2010, lo que ha provocado un aumento en la tasa de desempleo del 2,5\%, por lo cual la relación entre estas dos tasas ha sido de aproximadamente 2,5. En España, la situación ha sido más traumática, pues el crecimiento anual acumulativo experimentado en el PIBpc fue de $-1,9 \%$, mientras que la tasa de desempleo creció cerca del $12 \%$ en el mismo periodo, lo que ha provocado que la relación entre el crecimiento de la tasa de desempleo y la disminución del PIBpc sea de aproximadamente 6 , superando el doble de la relación experimentada por el conjunto de los países miembros de la UE-27.

Si comparamos entre los países miembros, se observa cómo algunos estados han experimentado reducciones del PIBpc superiores al de España, como Irlanda (-3,39\%), Grecia $(-3,11 \%)$, Italia $(-2,17 \%)$, 
PIBpc y tasa de desempleo en la Unión Europea

\begin{tabular}{|c|c|c|c|c|c|c|}
\hline & \multicolumn{3}{|c|}{ PIBpc (euros por habitantes) } & \multicolumn{3}{|c|}{ Tasa desempleo (\%) } \\
\hline & 2007 & 2010 & Cto. $(*)$ & 2007 & 2010 & Difer. \\
\hline Bélgica & 30.200 & 29.600 & $-0,67$ & 7,5 & 8,3 & 0,8 \\
\hline Bulgaria & 3.400 & 3.500 & 0,97 & 6,9 & 10,3 & 3,4 \\
\hline República Checa & 11.500 & 11.400 & $-0,29$ & - & 7,3 & $0,6(* *)$ \\
\hline Dinamarca & 39.900 & 37.300 & $-2,22$ & 3,8 & 7,5 & 3,7 \\
\hline Alemania & 29.000 & 29.100 & 0,11 & 8,7 & 7,1 & $-1,6$ \\
\hline Estonia & 9.900 & 8.400 & $-5,33$ & 4,6 & 16,9 & 12,3 \\
\hline Irlanda & 41.200 & 36.500 & $-3,96$ & 4,7 & 13,9 & 9,2 \\
\hline Grecia & 18.800 & 17.100 & $-3,11$ & 8,3 & 12,6 & 4,3 \\
\hline España & 21.800 & 20.600 & $-1,87$ & 8,3 & 20,1 & 11,8 \\
\hline Francia & 28.200 & 27.300 & $-1,08$ & 8,4 & 9,7 & 1,3 \\
\hline Italia & 25.100 & 23.500 & $-2,17$ & 6,1 & 8,4 & 2,3 \\
\hline Chipre & 19.400 & 18.500 & $-1,57$ & 4,1 & 6,5 & 2,4 \\
\hline Letonia & 7.200 & 5.900 & $-6,42$ & 6,5 & 19,8 & 13,3 \\
\hline Lituania & 7.400 & 7.100 & $-2,67$ & 3,8 & 18,0 & 14,2 \\
\hline Luxemburgo & 70.400 & 35.400 & $-2,43$ & 4,2 & 4,6 & 0,4 \\
\hline Hungría & 9.200 & 8.800 & $-1,47$ & 7,4 & 11,2 & 3,8 \\
\hline Malta & 12.900 & 13.200 & 0,77 & 6,5 & 6,9 & 0,4 \\
\hline Países Bajos & 33.700 & 33.100 & $-0,60$ & 3,6 & 4,5 & 0,9 \\
\hline Austria & 31.800 & 31.300 & $-0,53$ & 4,4 & 4,4 & 0,0 \\
\hline Polonia & 7.300 & 8.000 & 3,10 & 9,6 & 9,7 & 0,1 \\
\hline Portugal & 15.100 & 14.900 & $-0,44$ & 8,9 & 12,0 & 3,1 \\
\hline Rumanía & 4.200 & 4.200 & 0,00 & 6,4 & 7,3 & 0,9 \\
\hline Eslovenia & 16.100 & 15.300 & $-1,68$ & 4,9 & 7,3 & 2,4 \\
\hline Eslovaquia & 8.500 & 8.900 & 1,54 & 11,2 & 14,5 & 3,3 \\
\hline Finlandia & 32.700 & 30.600 & $-2,19$ & 6,9 & 8,4 & 1,5 \\
\hline Suecia & 35.100 & 34.500 & $-0,57$ & 6,1 & 8,6 & 2,5 \\
\hline Reino Unido & 32.200 & 30.500 & $-1,79$ & 5,3 & 7,8 & 2,5 \\
\hline Unión Europea (27) & 23.800 & 23.100 & $-0,99$ & 7,2 & 9,7 & 2,5 \\
\hline
\end{tabular}

${ }^{*}$ ) Tasa de crecimiento anual acumulativo en el periodo analizado.

(**) Diferencia entre 2009 y 2010.

PIBpc y tasa de desempleo en la Unión Europea

Fuente: elaboración de los autores, con base en datos de Eurostat e INE.

Luxemburgo $(-2,43 \%)$, Dinamarca $(-2,22 \%)$ y Finlandia $(-2,19 \%)$, en los que la relación existente entre el crecimiento de la tasa de paro y la reducción del PIBpc en ningún caso llega a duplicarse, con la excepción de Irlanda, donde dicha relación es algo superior a 2. Los países cuya relación entre las tasas de desempleo y de crecimiento del PIBpc se ha acercado más a la experimentada por la economía española han sido Lituania (con una caída del PIBpc de $2,67 \%$ y un crecimiento de la tasa de paro del $14,21 \%$ ) y Portugal (cuyo PIBpc desciende en 0,44 $\%$ y la tasa de paro creció en un 3,1\%). Por último, cabe señalar la posición de Alemania, que presentó un crecimiento del PIBpc del $0,11 \%$, y redujo así su tasa de desempleo en 1,6\%, siendo el único país de la Unión Europea que ha reducido su tasa de desempleo en el periodo analizado.

Todo lo anterior constata la fuerte desaceleración económica sufrida por la economía española a partir del segundo trimestre de 2008, mucho más intensa que la experimentada por la media de la UE-27, lo que provoca que la posición económica se vea retrasada en el ranking de los estados de la Unión Europea, y así se reafirman las limitaciones de su estructura productiva en el entorno europeo. 
IMPACTO DE LA CRISIS ECONÓMICA EN EL EMPLEO DE LAS REGIONES ESPAÑOLAS: UN ANÁLISIS SECTORIAL EN EL PERIODO 2007-2010

\section{ANÁLISIS REGIONAL DE LA CRISIS ECONÓMICA EN ESPAÑA}

La crisis económico-financiera ha afectado de forma muy desigual a las regiones españolas. Atendiendo a las tasas de crecimiento anual acumulativo del PIBpc y de las horas dedicadas a la actividad productiva, así como a las diferencias en tasas de desempleo (tabla 2), las regiones que han acusado con mayor intensidad los efectos de la crisis han sido Andalucía, Aragón Baleares, Canarias, Castilla-La Mancha, Comunidad Valenciana y Murcia, cuyos índices de crecimiento del PIBpc y horas trabajadas han presentado valores por debajo de la media regional, y sus tasas de desempleo se han situado por encima de la media del conjunto de las comunidades autónomas. El resto de las regiones españolas parece que ha soportado con mayor éxito la situación tan crítica que se viene padeciendo a nivel mundial.

Tabla 2.

\begin{tabular}{|c|c|c|c|c|c|c|c|c|c|}
\hline \multicolumn{10}{|c|}{ PIBpc, tasa de desempleo y número de horas trabajadas (2007-2010) } \\
\hline \multirow[b]{2}{*}{ Regiones } & \multicolumn{3}{|c|}{$\begin{array}{c}\text { PIBpc } \\
\text { (euros constantes 2000) }\end{array}$} & \multicolumn{3}{|c|}{$\begin{array}{c}\text { Tasa de desempleo } \\
(\%)\end{array}$} & \multicolumn{3}{|c|}{$\begin{array}{l}\text { Horas trabajadas } \\
\text { (millones horas) }\end{array}$} \\
\hline & 2007 & 2010 & Cto. $(*)$ & 2007 & 2010 & Difer. & 2007 & 2009 & Cto. $\left({ }^{*}\right)$ \\
\hline Andalucía & $13.048,5$ & $12.245,1$ & $-2,10$ & 12,76 & 27,97 & 15,21 & $6.027,65$ & $5.681,23$ & $-2,92$ \\
\hline Aragón & $19.220,0$ & $17.970,6$ & $-2,22$ & 5,23 & 14,78 & 9,54 & $1.250,48$ & $1.187,10$ & $-2,57$ \\
\hline Asturias & $16.314,2$ & $15.652,8$ & $-1,37$ & 8,48 & 15,97 & 7,49 & 851,52 & 818,67 & $-1,95$ \\
\hline Baleares & $15.858,5$ & $14.779,6$ & $-2,32$ & 6,98 & 20,37 & 13,39 & 968,75 & 920,75 & $-2,51$ \\
\hline Canarias & $15.059,3$ & $14.058,4$ & $-2,27$ & 10,44 & 28,70 & 18,26 & $1.660,11$ & $1.566,36$ & $-2,86$ \\
\hline Cantabria & $17.707,2$ & $16.869,5$ & $-1,60$ & 5,90 & 13,88 & 7,98 & 503,20 & 486,76 & $-1,65$ \\
\hline Castilla y León & $16.972,4$ & $16.350,6$ & $-1,24$ & 7,18 & 15,78 & 8,60 & $2.217,92$ & $2.117,91$ & $-2,28$ \\
\hline Castilla-La Mancha & $12.895,2$ & $11.937,9$ & $-2,54$ & 7,62 & 20,99 & 13,37 & $1.590,85$ & $1.527,48$ & $-2,01$ \\
\hline Cataluña & $20.064,9$ & $18.650,5$ & $-2,41$ & 6,55 & 17,76 & 11,21 & $6.686,94$ & $6.407,06$ & $-2,12$ \\
\hline C. Valenciana & $14.896,5$ & $13.979,6$ & $-2,10$ & 8,76 & 23,29 & 14,53 & $4.000,14$ & $3.724,35$ & $-3,51$ \\
\hline Extremadura & $12.073,0$ & $11.796,3$ & $-0,77$ & 13,07 & 23,06 & 10,00 & 830,67 & 812,71 & $-1,09$ \\
\hline Galicia & $14.461,9$ & $14.094,4$ & $-0,85$ & 7,63 & 15,40 & 7,76 & $2.234,57$ & $2.165,98$ & $-1,55$ \\
\hline Madrid & $23.629,8$ & $22.113,3$ & $-2,19$ & 6,30 & 16,08 & 9,78 & $6.314,25$ & $6.135,37$ & $-1,43$ \\
\hline Murcia & $14.111,1$ & $13.130,4$ & $-2,37$ & 7,57 & 23,35 & 15,78 & $1.126,73$ & $1.067,95$ & $-2,64$ \\
\hline Navarra & $22.242,9$ & $21.169,8$ & $-1,63$ & 4,77 & 11,84 & 7,07 & 633,45 & 604,93 & $-2,28$ \\
\hline País Vasco & $23.257,9$ & $22.589,0$ & $-0,97$ & 6,11 & 10,55 & 4,44 & 2031,57 & $1.943,38$ & $-2,19$ \\
\hline La Rioja & $19.174,1$ & $18.192,5$ & $-1,74$ & 5,66 & 14,27 & 8,61 & 289,26 & 272,80 & $-2,89$ \\
\hline España & $17.233,7$ & $16.245,1$ & $-1,95$ & 8,24 & 20,05 & 11,82 & $39.218,06$ & $37.440,77$ & $-2,29$ \\
\hline
\end{tabular}

(*) Tasa de crecimiento anual acumulativo en el periodo analizado.

Fuente: elaboración de los autores, con base en datos de Alcaide (2011) e INE. 
Además, resulta necesario destacar que entre las regiones que están siendo más castigadas por la desaceleración económica se encuentran Andalucía, Baleares, Canarias, Castilla-La Mancha, Comunidad Valenciana y Murcia, por ser regiones que en el periodo que se está examinando presentaban un nivel de desarrollo económico (medido a partir del PIBpc) inferior al de la media de las regiones españolas y unas tasas de desempleo superiores a la media regional.

Si se analiza el impacto de la crisis económica desde el punto de vista de la variación en el número de empresas según el número de asalariados, se observa un fenómeno de una enorme trascendencia, como es la destrucción generalizada de empresas con asalariados, siendo ésta mayor cuanto menor es el número de asalariados. Este hecho puede ser indicativo de la mayor resistencia de las grandes empresas a la crisis económica, al menos en sus primeros años, debido quizás a la fortaleza y estabilización de su estructura económica y financiera. En el periodo 2007-2010 se han cerrado 45.219 empresas, de las cuales 61.722 eran pequeñas empresas de 1 a 5 asalariados, 35.637 eran de 6 a 19 asalariados y 11.944 eran de 20 a 49 asalariados. También resulta interesante poner de manifiesto el cierre de 1994 empresas con un número de empleados comprendido entre 50 y 99, 1524 con un número de empleados entre 100 y 499 y 151 empresas con más de 500 empleados. Así mismo, sorprende la creación de 67.753 empresas sin asalariados en un periodo de aguda crisis económica, lo que podría ser explicado por la consideración que dicho tipo de empresa ha tenido, en muchos casos, como ocupación refugio o como socorro de muchos de los trabajadores que estaban perdiendo su empleo en el periodo analizado 6 .

Tabla 3.

Variación en el número de empresas según asalariados (número de empresas) (2007-2010)

\begin{tabular}{|c|c|c|c|c|c|c|c|c|}
\hline Regiones & Sin asal. & $1-5$ & $6-19$ & $20-49$ & $50-99$ & $100-499$ & Más 500 & TOTAL \\
\hline Andalucía & 3.127 & -5.800 & -7.350 & -2.312 & -471 & -326 & -17 & -13.149 \\
\hline Aragón & 1.819 & -949 & -506 & -277 & 11 & -49 & -6 & 43 \\
\hline Asturias & -184 & -1.185 & -360 & -146 & -13 & -21 & -5 & -1.914 \\
\hline Baleares & 1.857 & -1.988 & -1.125 & -348 & -21 & -53 & -14 & -1.692 \\
\hline Canarias & 2.706 & -4.035 & -2.029 & -818 & -130 & -133 & -21 & -4.460 \\
\hline Cantabria & 416 & -472 & -336 & -112 & -29 & -3 & 0 & -536 \\
\hline Castilla y León & 1.824 & -1.782 & -942 & -346 & -53 & -52 & 4 & -1.347 \\
\hline Castilla-La Mancha & 2.305 & -1.410 & -1.329 & -512 & -91 & -33 & 0 & -1.070 \\
\hline Cataluña & 19.717 & -12.461 & -7.323 & -2.114 & -346 & -202 & -5 & -2.734 \\
\hline C. Valenciana & 4.310 & -11.354 & -6.619 & -2.003 & -352 & -193 & -9 & -16.220 \\
\hline Extremadura & 1.164 & -1.348 & -331 & -126 & -19 & 4 & -3 & -659 \\
\hline Galicia & 3.141 & -2.628 & -1.214 & -373 & -26 & -32 & -14 & -1.146 \\
\hline Madrid & 17.553 & -11.871 & -3.290 & -1.299 & -223 & -327 & -42 & 501 \\
\hline Murcia & 784 & -3.002 & -2.145 & -634 & -126 & -39 & -16 & -5.178 \\
\hline Navarra & -7 & -397 & -253 & -84 & -36 & -15 & -3 & -795 \\
\hline País Vasco & 6.965 & -826 & -350 & -345 & -51 & -38 & -4 & 5.351 \\
\hline La Rioja & 256 & -214 & -135 & -95 & -18 & -12 & 4 & -214 \\
\hline España & 67.753 & -61.722 & -35.637 & -11.944 & -1.994 & -1.524 & -151 & -45.219 \\
\hline
\end{tabular}

Fuente: elaboración de los autores, con base en datos de INE.

$6 \quad$ Resulta conveniente tener en cuenta que la influencia del mercado monetario en la estructura económica y financiera de las empresas no deja de ser un aspecto relevante a la hora de explicar la resistencia que muestran algunas empresas a reducir los contratos. 
IMPACTO DE LA CRISIS ECONÓMICA EN EL EMPLEO DE LAS REGIONES ESPAÑOLAS: UN ANÁLISIS SECTORIAL EN EL PERIODO 2007-2010

Así mismo, se observa cómo algunas regiones crean un importante volumen de empresas en términos netos, como Cataluña y Madrid (por las empresas creadas sin asalariados, que supone un $55 \%$ del total de empresas creadas), y La Rioja y Castilla y León (con una creación de cuatro empresas con más de 500 empleados cada una), las cuales se destacan como regiones con un importante espíritu emprendedor. Por otro lado, resulta conveniente señalar la existencia de regiones que experimentan un importante volumen de pérdidas de empresas con asalariados, como la Comunidad Valenciana, Andalucía, Murcia y Canarias, que destacan por la pérdida de pequeñas y medianas empresas, y de grandes sociedades.

Tabla 4.

Número de empleos (2007-2010)

\begin{tabular}{|c|c|c|c|c|c|c|c|c|c|}
\hline \multirow[b]{2}{*}{ Regiones } & \multicolumn{3}{|c|}{$\begin{array}{c}\text { Empleos totales } \\
\text { (número de personas) }\end{array}$} & \multicolumn{3}{|c|}{$\begin{array}{l}\text { Empleos asalariados } \\
\text { (número de personas) }\end{array}$} & \multicolumn{3}{|c|}{$\begin{array}{l}\text { Empleos no asalariados } \\
\text { (número de personas) }\end{array}$} \\
\hline & 2007 & 2010 & Cto. $\left({ }^{*}\right)$ & 2007 & 2010 & Cto. $\left({ }^{*}\right)$ & 2007 & 2010 & Cto. $\left({ }^{*}\right)$ \\
\hline Andalucía & 3.055 .181 & 2.715 .661 & $-3,85$ & 2.499 .712 & 2.196 .103 & $-4,22$ & 555.469 & 519.558 & $-2,20$ \\
\hline Aragón & 603.830 & 554.437 & $-2,80$ & 478.767 & 439.219 & $-2,83$ & 125.063 & 115.218 & $-2,70$ \\
\hline Asturias & 441.220 & 417.043 & $-1,86$ & 336.639 & 320.687 & $-1,61$ & 104.581 & 96.356 & $-2,69$ \\
\hline Baleares & 522.683 & 467.494 & $-3,65$ & 425.923 & 376.519 & $-4,03$ & 96.760 & 90.975 & $-2,03$ \\
\hline Canarias & 878.874 & 749.312 & $-5,18$ & 751.815 & 631.520 & $-5,65$ & 127.059 & 117.792 & $-2,49$ \\
\hline Cantabria & 242.357 & 224.394 & $-2,53$ & 186.962 & 172.236 & $-2,70$ & 55.395 & 52.158 & $-1,99$ \\
\hline Castilla y León & 1.066 .685 & 1.001 .194 & $-2,09$ & 780.199 & 732.680 & $-2,07$ & 286.486 & 268.514 & $-2,14$ \\
\hline Castilla-La Mancha & 817.538 & 733.522 & $-3,55$ & 616.732 & 545.348 & $-4,02$ & 200.806 & 188.174 & $-2,14$ \\
\hline Cataluña & 3.287 .834 & 2.942 .444 & $-3,63$ & 2.755 .139 & 2.456 .705 & $-3,75$ & 532.695 & 485.739 & $-3,03$ \\
\hline C. Valenciana & 2.061 .939 & 1.759 .720 & $-5,15$ & 1.701 .772 & 1.432 .715 & $-5,58$ & 360.167 & 327.005 & $-3,17$ \\
\hline Extremadura & 428.292 & 404.805 & $-1,86$ & 316.012 & 294.402 & $-2,33$ & 112.280 & 110.403 & $-0,56$ \\
\hline Galicia & 1.201 .717 & 1.135 .055 & $-1,88$ & 800.838 & 760.996 & $-1,69$ & 400.879 & 374.059 & $-2,28$ \\
\hline Madrid & 2.975 .860 & 2.788 .441 & $-2,15$ & 2.627 .767 & 2.458 .127 & $-2,20$ & 348.093 & 330.314 & $-1,73$ \\
\hline Murcia & 578.659 & 500.849 & $-4,70$ & 486.637 & 417.314 & $-4,99$ & 92.022 & 83.535 & $-3,17$ \\
\hline Navarra & 281.776 & 265.440 & $-1,97$ & 229.214 & 216.752 & $-1,85$ & 52.562 & 48.688 & $-2,52$ \\
\hline País Vasco & 999.212 & 941.615 & $-1,96$ & 830.116 & 781.838 & $-1,98$ & 169.096 & 159.777 & $-1,87$ \\
\hline La Rioja & 151.616 & 139.381 & $-2,77$ & 117.977 & 107.379 & $-3,09$ & 33.639 & 32.002 & $-1,65$ \\
\hline España & 19.595 .273 & 17.740 .807 & $-3,26$ & 15.942 .221 & 14.340 .540 & $-3,47$ & 3.653 .052 & 3.400 .267 & $-2,36$ \\
\hline
\end{tabular}

${ }^{*}$ ) Tasa de crecimiento anual acumulativo en el periodo analizado.

Fuente: elaboración de los autores, con base en datos de Alcaide (2011). 
Si se realiza una valoración del efecto de la crisis económica en las regiones españolas en términos de pérdidas de empleo (tabla 4), se pueden realizar las siguientes consideraciones. Por un lado, que la pérdida de empleos ha sido generalizada en todas las regiones españolas, tanto en los empleos asalariados como en los no asalariados, aunque no con la misma fuerza en todas ellas. De hecho, las regiones cuyas tasas de crecimiento del empleo han sido más desfavorables que la media regional española (cifrada en una pérdida de empleos del 3,26\% anual acumulativo) son Canarias $(-5,18 \%)$, Comunidad Valenciana $(-5,15 \%)$, Murcia $(-4,70 \%)$, Andalucía $(-3,85 \%)$, Baleares $(-3,65 \%)$, Cataluña $(-3,63 \%)$ y Castilla-La Mancha $(-3,55 \%)$. Son todas estas regiones precisamente las que han experimentado unas tasas de destrucción de empleos asalariados superiores a la media regional española, no así cuando nos referimos a los empleos no asalariados, en los cuales de las regiones referidas anteriormente sólo presentan crecimientos inferiores a la media regional Canarias $(-2,49 \%)$, Cataluña $(-3,03 \%)$, Comunidad Valenciana y Murcia $(-3,17 \%)$.

El examen de la evolución de las tasas de desempleo por niveles de formación en el periodo analizado (tabla 5) permite aportar algunas ideas muy interesantes sobre los objetivos marcados en la presente investigación. Por un lado, resulta necesario destacar que el incremento de la tasa de desempleo padecida por la economía española $(11,83 \%)$ ha afectado de manera muy dispar a la población, según el nivel de formación y estudios que posee. En efecto, el crecimiento de la tasa de paro ha sido más intenso en el colectivo de población analfabeta, sin estudios y con solo estudios primarios, que creció un $19,74 \%$. Sin embargo, los crecimientos experimentados por

Tabla 5.

Variación de las tasas de desempleo por niveles de formación (2007-2010)

\begin{tabular}{|c|c|c|c|c|c|}
\hline Regiones & 1 & 2 & 3 & 4 & 5 \\
\hline Andalucía & 23,97 & 17,39 & 12,10 & 5,06 & 15,02 \\
\hline Aragón & 11,75 & 10,97 & 9,38 & 3,66 & 8,96 \\
\hline Asturias & 9,05 & 11,68 & 5,23 & 4,91 & 7,90 \\
\hline Baleares & 18,52 & 13,97 & 12,20 & 6,75 & 12,96 \\
\hline Canarias & 27,20 & 23,00 & 16,34 & 3,47 & 19,04 \\
\hline Cantabria & 17,40 & 9,79 & 6,22 & 3,44 & 7,99 \\
\hline Castilla y León & 15,12 & 11,90 & 6,95 & 3,56 & 9,14 \\
\hline Castilla-La Mancha & 23,09 & 14,94 & 10,85 & 5,19 & 13,74 \\
\hline Cataluña & 16,78 & 15,31 & 7,26 & 4,50 & 11,16 \\
\hline C. Valenciana & 24,16 & 17,17 & 13,98 & 5,29 & 15,07 \\
\hline Extremadura & 8,30 & 12,58 & 9,30 & 1,32 & 9,37 \\
\hline Galicia & 11,14 & 10,14 & 7,45 & 2,74 & 7,94 \\
\hline Madrid & 20,34 & 13,90 & 10,25 & 4,37 & 10,13 \\
\hline Murcia & 18,04 & 15,50 & 12,40 & 5,50 & 13,71 \\
\hline Navarra & 18,16 & 6,42 & 5,35 & 3,55 & 6,20 \\
\hline País Vasco & 8,94 & 5,98 & 5,12 & 0,71 & 4,29 \\
\hline La Rioja & 9,47 & 10,80 & 6,63 & $-0,25$ & 6,53 \\
\hline España & 19,74 & 14,77 & 9,96 & 4,16 & 11,83 \\
\hline
\end{tabular}

1. Analfabetos, sin estudios y primarios.

2. Bachiller elemental/EGB/ESO, formación profesional l/ciclos formativos de grado medio.

3. Bachiller Superior/BUP y COU/ Bachillerato, Formación Profesional II/Ciclos Formativos de Grado Superior.

4. Estudios anteriores al superior y estudios superiores.

5. Total nivel de estudios terminados.

Fuente: elaboración de los autores, con base en datos de IVIE. 
los segmentos de población con estudios fueron menos significativos: la población con estudios de bachiller elemental, EGB, ESO, formación profesional I y ciclos formativos de grado medio vieron aumentada su tasa de paro en 14,77 puntos; la población con formación y estudios de bachiller superior, BUP, COU, bachillerato, formación profesional II y ciclos formativos de grado superior experimentó un incremento de 9,96\% en su tasa de desempleo; por último, el segmento de población con mayor formación y estudios (anteriores al superior y estudios superiores) solo sufrió una subida de la tasa de desempleo del 4,16\%. Lo anterior nos permite constatar que la crisis económica ha afectado a todos los segmentos de población según el nivel de estudios, pero el grado de incidencia ha sido destacadamente mayor en los trabajadores con menor nivel de formación.

Por otro lado, resulta conveniente poner de manifiesto que las comunidades autónomas que han acusado la crisis con mayor énfasis según el nivel de estudios que presenta su población han sido las siguientes: en el primer grupo (analfabetos, sin estudios y con estudios primarios), Canarias, Comunidad Valenciana, Andalucía, Castilla-La Mancha y Madrid; en el segundo grupo (bachiller elemental/EGB/ESO, formación profesional I/ciclos formativos de grado medio), Canarias, Andalucía, Comunidad Valenciana, Murcia, Cataluña y Castilla-La Mancha; en el tercer grupo (bachiller superior/BUP y COU/ bachillerato, formación profesional II/ciclos formativos de grado superior), Canarias, Comunidad Valenciana, Murcia, Baleares, Andalucía, Castilla-La Mancha y Madrid; y en el cuarto grupo (estudios anteriores al superior y estudios superiores), Baleares, Murcia, Comunidad Valenciana, Castilla-La Mancha, Andalucía, Asturias, Cataluña y Madrid.

\section{IMPACTO SECTORIAL DE LA CRISIS ECONÓMICA EN LAS REGIONES ESPAÑOLAS}

Este apartado trata de analizar cómo ha afectado la crisis económica a los distintos sectores económicos de cada una de las regiones españolas. Según los datos de la tabla 6, la economía española ha perdido 1,8 millones de empleos entre 2007 y 2010 . De estos, el 46,0 \% corresponden a los empleos del sector de la construcción. Efectivamente, este sector, considerado como el motor del crecimiento económico durante los años 90 y hasta 2008, fue el más afectado por la crisis económica. Siendo especialmente intensivo en mano de obra, alcanzó en la fase de expansión niveles muy elevados, y en el periodo de crisis económica ha experimentado una intensa caída de la actividad que se ha traducido en una destrucción de empleos muy significativa (Urtasun et al., 2012). Le siguen, por orden de importancia, el sector de servicios privados, cuya pérdida de empleos fue de 568 mil, lo que supone el 30,6 \% de los empleos perdidos en la economía, y el sector industrial, con una pérdida de 525 mil empleos, lo que supuso un $28,3 \%$ del total de pérdidas de empleo. Ello, sin duda, hace que la crisis económica haya afectado con especial virulencia a un sector que históricamente ha presentado un mayor nivel de productividad del empleo, como es el sector manufacturero, lo que sin duda debilita y pone en entredicho el modelo productivo en España.

Si se analiza por regiones, la pérdida de empleos ha sido más intensa en algunas regiones como Cataluña (con una pérdida de 18,6\%), Andalucía (con una pérdida de 18,31\%) y Comunidad Valenciana (con una pérdida de 16,3\%). A estas regiones le siguieron, por orden de importancia en la disminución de empleos, Madrid, Canarias, Castilla-La Mancha, Murcia, Galicia y Castilla y León. Resulta interesante destacar también el aumento de empleos que experimentan el sector servicios públicos y el sector energía y agua en el periodo de crisis económica que se viene analizando. 
Antonio Rafael Peña Sánchez • Mercedes Jiménez García • José Ruiz Chico

Tabla 6.

Variación de empleos (2007-2010)

(número de empleos)

\begin{tabular}{|c|c|c|c|c|c|c|c|}
\hline Regiones & Agr. y Pesca & $\begin{array}{l}\text { Agua y } \\
\text { Energ. }\end{array}$ & Indust. & Constr. & Serv. Priv. & Serv. Púb. & TOTAL \\
\hline Andalucía & -75.229 & 10.745 & -49.181 & -179.636 & -87.195 & 40.976 & -339.520 \\
\hline Aragón & -6.331 & 2.670 & -19.598 & -17.505 & -16.971 & 8.342 & -49.393 \\
\hline Asturias & -4.460 & 5.761 & -6.925 & -14.240 & -8.923 & 4.610 & -24.177 \\
\hline Baleares & -1.532 & 1.589 & -6.537 & -20.597 & -30.752 & 2.640 & -55.189 \\
\hline Canarias & -928 & 2.933 & -9.211 & -54.235 & -68.555 & 434 & -129.562 \\
\hline Cantabria & -3.906 & 1.374 & -4.638 & -10.376 & -3.585 & 3.168 & -17.963 \\
\hline Castilla y León & -16.121 & 8.832 & -22.568 & -43.427 & -8.851 & 16.644 & -65.491 \\
\hline Castilla-La Mancha & -13.086 & 3.650 & -23.150 & -48.376 & -15.860 & 12.806 & -84.016 \\
\hline Cataluña & -10.502 & 9.886 & -142.637 & -124.157 & -111.287 & 33.307 & -345.390 \\
\hline C. Valenciana & -10.821 & 5.722 & -92.163 & -111.566 & -112.773 & 19.382 & -302.219 \\
\hline Extremadura & -10.974 & 1.889 & -4.447 & -17.617 & -112 & 7.774 & -23.487 \\
\hline Galicia & -16.669 & 5.958 & -26.276 & -36.093 & -4.751 & 11.169 & -66.662 \\
\hline Madrid & 1.402 & 10.894 & -53.165 & -105.183 & -59.315 & 17.948 & -187.419 \\
\hline Murcia & -10.003 & 1.754 & -15.074 & -38.976 & -22.095 & 6.584 & -77.810 \\
\hline Navarra & -2.658 & 848 & -8.105 & -8.439 & -764 & 2.782 & -16.336 \\
\hline País Vasco & -3.612 & 3.628 & -36.214 & -19.574 & -14.521 & 12.696 & -57.597 \\
\hline La Rioja & -3.541 & 323 & -5.353 & -3.813 & -1.823 & 1.972 & -12.235 \\
\hline España & -188.971 & 78.456 & -525.242 & -853.810 & -568.133 & 203.234 & -1.854 .466 \\
\hline
\end{tabular}

Fuente: elaboración de los autores, con base en datos de Alcaide (2011). 
Por sectores productivos, se observa en la agricultura y pesca una fuerte reducción de empleos en Andalucía (75.229 puestos de trabajo), que supone prácticamente el $40 \%$ de total de empleos perdidos en el sector. En esta misma actividad, resulta interesante resaltar a otras regiones que sufren una importante destrucción de mano de obra como Galicia (16.669), Castilla y León (16.121) y Castilla-La Mancha (13.086).

Teniendo en cuenta la grave situación económica que está padeciendo la economía española desde el año 2008, en el sector agua y energía se produce un elevado incremento del empleo, de 78.456 puestos. Entre las regiones con mayor aumento del empleo en este sector productivo se encuentran Madrid (10.894), Andalucía (10.745), Cataluña (9.886) y Castilla y León (8.832).

El sector industrial ha sido uno de los más castigados en la crisis económica. Cataluña (con una pérdida de ocupación de 142.637 empleos), Comunidad Valenciana (92.163), Madrid (53.165), Andalucía (49.181) y País Vasco (36.214) conforman las regiones con mayor destrucción de empleos en el periodo analizado.

El sector de la construcción ha sido el más azotado por la situación de deterioro económico generalizado. Las regiones españolas más representativas por la evolución de su nivel de empleo han sido Andalucía (que pierde 179.636 empleos), Cataluña (124.157), Comunidad Valenciana (111.566) y Madrid (105.183).

Otro sector muy afectado por la situación económica vivida a partir de 2008 en la economía española ha sido el sector servicios privados, en el que las regiones más distintivas han sido la Comunidad Valenciana (que sufre una reducción de 112.773 empleos), Cataluña (111.287), Andalucía (87.195), Canarias (68.555) y Madrid (59.315).

Por último, el sector servicios públicos ha creado en el periodo analizado 203.234 puestos de trabajo, siendo las regiones más favorecidas Andalucía (con una creación de 40.976 puestos de trabajo), Cataluña (33.307), Comunidad Valenciana (19.382), Madrid (17.948) y Castilla y León (16.644).
Del análisis anterior se deduce que la caída del empleo ha sido una constante en prácticamente todos los sectores productivos, con la excepción del sector servicios públicos o servicios no destinados a la venta y el sector agua y energía. Por ende, los sectores más afectados por la crisis económica han sido la construcción, el sector servicios privados o servicios destinados a la venta y la industria. Además, las regiones que han tenido que soportar con mayor virulencia los efectos del shock han sido Andalucía, Cataluña, Comunidad Valenciana y Madrid. Teniendo en cuenta que Andalucía se posiciona entre las regiones con menor nivel de desarrollo económico en España, parece que la crisis puede estar debilitándola aún más económicamente, lo que la sitúa en un estado muy delicado en el conjunto de las regiones españolas?.

La explicación anterior se contrasta en las tablas 7 y 8, donde se presenta la variación de la población ocupada por regiones y sectores económicos según nivel de formación o estudios, para el periodo 2007-2010. Como se expuso anteriormente, el sector construcción, los servicios destinados a la venta y la industria se han convertido en los sectores más perjudicados por la situación en la que se encuentra la economía española. De las tablas referenciadas se puede extraer también que, por lo general, la pérdida de empleos en prácticamente todos los sectores se produce principalmente en los segmentos de ocupación con menor nivel de formación y estudios. Es más, en los sectores en los que se produce un crecimiento del empleo, este último se pone de manifiesto principalmente en aquella parte de población con mayor nivel de formación y estudios.

7 En este sentido, resulta necesario indicar que sería interesante profundizar en la investigación sobre los mecanismos de transmisión de la crisis a las regiones españolas, estableciendo el impacto de los cambios producidos en el mercado monetario sobre el mercado de bienes y servicios $y$, por tanto, en el empleo. No obstante, debido a que este análisis trasciende del objeto de estudio de este trabajo, se ha decidido centrarlo en el análisis de la influencia de la crisis sobre el empleo de las regiones españolas y dejar el impacto del mercado monetario para una futura investigación. 
Antonio Rafael Peña Sánchez • Mercedes Jiménez García • José Ruiz Chico

Tabla 7.

Variación de población ocupada por regiones, sectores económicos y nivel de formación y estudios (2007-2010) (miles de empleos)

\begin{tabular}{|c|c|c|c|c|c|c|c|c|c|c|c|c|c|c|c|}
\hline \multirow[b]{2}{*}{ Regiones } & \multicolumn{5}{|c|}{ Agricultura } & \multicolumn{5}{|c|}{ Energía } & \multicolumn{5}{|c|}{ Industria } \\
\hline & 1 & 2 & 3 & 4 & 5 & 1 & 2 & 3 & 4 & 5 & 1 & 2 & 3 & 4 & 5 \\
\hline Andalucía & $-29,73$ & 0,50 & 1,15 & $-5,23$ & $-33,31$ & $-0,40$ & 3,58 & 3,07 & 1,08 & 7,33 & $-32,20$ & $-40,92$ & $-3,25$ & 3,81 & $-72,56$ \\
\hline Aragón & 0,05 & $-0,43$ & 0,78 & 0,37 & 0,77 & $-0,32$ & 0,03 & 0,47 & 0,86 & 1,04 & $-5,76$ & $-11,39$ & $-2,65$ & $-4,79$ & $-24,59$ \\
\hline Asturias & $-0,79$ & $-4,10$ & 0,46 & 0,52 & $-3,91$ & $-0,68$ & $-1,02$ & $-1,01$ & 0,63 & $-2,08$ & $-4,89$ & $-5,07$ & $-0,91$ & $-2,78$ & $-13,65$ \\
\hline Baleares & 0,09 & 0,14 & $-0,05$ & $-0,49$ & $-0,31$ & $-0,79$ & 0,79 & 0,93 & 0,33 & 1,26 & $-3,05$ & $-6,94$ & $-0,45$ & $-3,56$ & $-14,00$ \\
\hline Canarias & 3,03 & $-7,08$ & 1,34 & $-0,44$ & $-3,15$ & $-0,45$ & $-0,37$ & $-2,01$ & 0,02 & $-2,81$ & $-5,71$ & $-2,00$ & $-0,87$ & 0,83 & $-7,75$ \\
\hline Cantabria & $-2,38$ & 1,93 & 0,51 & $-0,72$ & $-0,67$ & 0,15 & $-0,03$ & 0,20 & 0,16 & 0,48 & $-2,34$ & $-2,24$ & $-3,04$ & $-1,82$ & $-9,44$ \\
\hline $\begin{array}{l}\text { Castilla y } \\
\text { León }\end{array}$ & $-10,89$ & $-3,13$ & 1,46 & $-1,92$ & $-14,48$ & 0,33 & $-0,11$ & $-1,27$ & 0,88 & $-0,17$ & $-9,67$ & $-13,91$ & $-3,53$ & 1,02 & $-26,09$ \\
\hline $\begin{array}{c}\text { Castilla-La } \\
\text { Mancha }\end{array}$ & $-7,86$ & 0,15 & $-0,42$ & 0,65 & $-7,48$ & $-0,45$ & $-0,89$ & $-0,76$ & $-0,15$ & $-2,26$ & $-6,30$ & $-16,14$ & 2,14 & $-0,27$ & $-20,57$ \\
\hline Cataluña & 0,72 & $-7,24$ & 11,42 & 1,53 & 6,43 & $-0,50$ & $-3,92$ & 0,78 & $-3,08$ & $-6,72$ & $-21,01$ & $-91,05$ & $-28,54$ & $-12,62$ & $-153,22$ \\
\hline $\begin{array}{c}\text { C. } \\
\text { Valenciana }\end{array}$ & $-1,90$ & $-2,53$ & $-5,84$ & $-3,12$ & $-13,38$ & 0,94 & 1,78 & $-0,37$ & $-0,25$ & 2,10 & $-15,34$ & $-76,56$ & $-40,92$ & 0,69 & $-132,13$ \\
\hline Extremadura & $-2,35$ & $-0,70$ & $-0,53$ & 1,16 & $-2,43$ & 0,14 & $-0,04$ & $-0,26$ & 0,29 & 0,13 & $-0,95$ & $-7,21$ & 3,36 & $-1,04$ & $-5,85$ \\
\hline Galicia & $-15,00$ & 4,61 & $-5,20$ & 0,90 & $-14,69$ & $-0,78$ & $-0,05$ & 0,32 & 0,99 & 0,48 & $-14,84$ & $-21,15$ & $-4,25$ & $-1,94$ & $-42,18$ \\
\hline Madrid & $-1,46$ & $-5,29$ & $-4,97$ & $-1,34$ & $-13,06$ & 0,26 & 2,62 & $-0,50$ & 9,28 & 11,66 & $-6,57$ & $-25,66$ & $-29,54$ & $-6,73$ & $-68,50$ \\
\hline Murcia & 7,90 & 7,78 & 2,05 & $-2,00$ & 15,73 & 0,02 & 0,52 & 0,17 & 0,48 & 1,18 & $-6,26$ & $-12,75$ & $-6,48$ & $-5,26$ & $-30,74$ \\
\hline Navarra & 0,60 & $-0,28$ & $-0,51$ & $-0,42$ & $-0,61$ & 0,11 & 0,20 & 0,39 & 0,08 & 0,79 & $-3,82$ & $-1,42$ & 0,58 & 0,89 & $-3,76$ \\
\hline País Vasco & $-0,31$ & 0,31 & $-0,68$ & $-0,43$ & $-1,10$ & $-0,05$ & $-0,42$ & $-2,00$ & 2,84 & 0,38 & $-11,60$ & $-2,15$ & $-12,52$ & $-2,93$ & $-29,20$ \\
\hline La Rioja & $-1,94$ & 0,30 & 0,53 & $-0,05$ & $-1,16$ & $-0,06$ & $-0,29$ & 0,04 & 0,08 & $-0,23$ & $-3,23$ & 0,75 & $-2,75$ & 2,44 & $-2,79$ \\
\hline España & $-62,20$ & $-15,06$ & 1,51 & $-11,03$ & $-86,78$ & $-2,54$ & 2,37 & $-1,80$ & 14,52 & 12,55 & $-153,53$ & $-335,78$ & $-133,62$ & $-34,07$ & $-657,00$ \\
\hline
\end{tabular}

1. Analfabetos, sin estudios y primarios.

2. Bachiller elemental/EGB/ESO, formación profesional I/ciclos formativos de grado medio.

3. Bachiller superior/BUP y COU/ bachillerato, formación profesional II/ciclos formativos de grado superior.

4. Estudios anteriores al superior y estudios superiores.

5. Total nivel de estudios terminados.

Fuente: elaboración de los autores, con base en datos de IVIE. 
IMPACTO DE LA CRISIS ECONÓMICA EN EL EMPLEO DE LAS REGIONES ESPAÑOLAS: UN ANÁLISIS SECTORIAL EN EL PERIODO 2007-2010

Tabla 8.

Variación de población ocupada por regiones, sectores económicos y nivel de formación y estudios (2007-2010) (miles de empleos)

\begin{tabular}{|c|c|c|c|c|c|c|c|c|c|c|c|c|c|c|c|}
\hline & \multicolumn{5}{|c|}{ Construcción } & \multicolumn{5}{|c|}{ Servicios de venta } & \multicolumn{5}{|c|}{ Servicios de no venta } \\
\hline Regiones & 1 & 2 & 3 & 4 & 5 & 1 & 2 & 3 & 4 & 5 & 1 & 2 & 3 & 4 & 5 \\
\hline Andalucía & $-87,91$ & $-133,80$ & $-11,76$ & $-6,97$ & $-240,43$ & $-44,33$ & $-26,06$ & $-11,74$ & 9,79 & $-72,34$ & 4,35 & 30,98 & 8,36 & 18,49 & 62,18 \\
\hline Aragón & $-4,80$ & $-7,76$ & $-2,88$ & 2,34 & $-13,10$ & $-1,77$ & $-8,41$ & $-0,48$ & $-12,44$ & $-23,11$ & 0,06 & 3,40 & 4,37 & $-5,88$ & 1,95 \\
\hline Asturias & $-2,36$ & $-4,41$ & $-5,93$ & $-0,38$ & $-13,07$ & $-7,65$ & 2,28 & 2,44 & 4,86 & 1,93 & $-0,32$ & $-0,71$ & $-2,68$ & 2,65 & $-1,06$ \\
\hline Baleares & $-11,84$ & $-10,85$ & 0,53 & $-1,88$ & $-24,03$ & $-1,69$ & $-5,39$ & 9,16 & $-2,48$ & $-0,40$ & 1,51 & 2,11 & 4,10 & 6,38 & 14,10 \\
\hline Canarias & $-24,44$ & $-32,59$ & $-13,22$ & $-5,16$ & $-75,41$ & $-15,50$ & $-19,21$ & $-13,88$ & $-13,26$ & $-61,84$ & $-5,26$ & $-5,08$ & 5,59 & $-0,69$ & $-5,44$ \\
\hline Cantabria & $-4,73$ & $-5,57$ & $-1,67$ & 1,58 & $-10,39$ & $-4,72$ & $-0,94$ & 1,47 & 3,31 & $-0,88$ & 0,57 & 0,83 & $-0,35$ & 2,61 & 3,65 \\
\hline Castilla y León & $-15,72$ & $-20,01$ & $-4,69$ & 0,37 & $-40,06$ & $-13,57$ & 8,61 & $-11,69$ & 0,08 & $-16,57$ & $-0,86$ & 5,85 & 0,18 & 14,20 & 19,37 \\
\hline $\begin{array}{c}\text { Castilla-La } \\
\text { Mancha }\end{array}$ & $-21,48$ & $-21,95$ & $-8,18$ & $-2,63$ & $-54,24$ & $-17,11$ & 9,91 & 0,29 & 7,77 & 0,86 & $-0,60$ & 6,46 & 6,37 & 0,28 & 12,51 \\
\hline Cataluña & $-46,10$ & $-69,84$ & $-27,67$ & $-11,81$ & $-155,41$ & 11,64 & $-88,33$ & $-35,54$ & 4,93 & $-107,30$ & 7,06 & $-4,10$ & 26,83 & 17,38 & 47,18 \\
\hline C. Valenciana & $-36,18$ & $-71,80$ & $-29,34$ & $-6,62$ & $-143,94$ & 0,57 & $-33,10$ & $-0,99$ & 1,96 & $-31,56$ & 0,95 & $-4,16$ & 8,75 & 15,82 & 21,35 \\
\hline Extremadura & $-2,79$ & $-13,31$ & $-0,31$ & $-0,27$ & $-16,68$ & $-3,08$ & 3,22 & $-3,74$ & 0,55 & $-3,05$ & $-2,23$ & 6,88 & 0,32 & $-1,12$ & 3,85 \\
\hline Galicia & $-16,47$ & $-21,12$ & $-5,60$ & $-1,15$ & $-44,34$ & $-25,44$ & 26,58 & $-10,99$ & 5,16 & $-4,70$ & $-3,57$ & 4,05 & 3,13 & 2,12 & 5,73 \\
\hline Madrid & $-35,91$ & $-45,51$ & $-31,99$ & 0,85 & $-112,55$ & $-34,67$ & $-19,32$ & $-3,89$ & 26,14 & $-31,74$ & $-10,08$ & 8,41 & $-7,33$ & 39,09 & 30,09 \\
\hline Murcia & $-15,58$ & $-20,87$ & $-8,59$ & $-1,60$ & $-46,65$ & 3,39 & 1,05 & $-8,32$ & $-3,61$ & $-7,48$ & 0,59 & 0,25 & $-0,06$ & 12,74 & 13,52 \\
\hline Navarra & $-4,89$ & $-4,84$ & $-3,18$ & $-0,74$ & $-13,64$ & $-6,52$ & 5,57 & 4,80 & 1,31 & 5,16 & $-0,67$ & $-1,91$ & 1,05 & 1,05 & $-0,49$ \\
\hline País Vasco & $-8,32$ & $-9,40$ & $-2,33$ & $-0,37$ & $-20,43$ & $-13,17$ & 13,17 & $-14,12$ & 20,33 & 6,20 & $-1,11$ & 1,16 & $-4,49$ & 3,73 & $-0,70$ \\
\hline La Rioja & $-3,24$ & $-1,95$ & 0,63 & 0,35 & $-4,22$ & $-3,82$ & 0,76 & $-0,01$ & 4,03 & 0,96 & 0,31 & $-0,05$ & 0,69 & $-1,64$ & $-0,69$ \\
\hline España & $-342,74$ & $-495,57$ & $-156,18$ & $-34,09$ & $-1.028,58$ & $-177,42$ & $-129,63$ & $-97,24$ & 58,42 & $-345,87$ & $-9,29$ & 54,35 & 54,82 & 127,20 & 227,08 \\
\hline
\end{tabular}

1.- Analfabetos, sin estudios y primarios.

2.- Bachiller elemental/EGB/ESO, formación profesional I/ciclos formativos de grado medio.

3.- Bachiller superior/BUP y COU/ bachillerato, formación profesional II/ciclos formativos de grado superior.

4.- Estudios anteriores al superior y estudios superiores.

5.- Total nivel de estudios terminados.

Fuente: elaboración de los autores, con base en datos de IVIE. 
Por sectores y regiones, el sector agrario pierde una importante cantidad de empleos en Andalucía, Castilla y León, Comunidad Valenciana, Galicia y Madrid, donde la mayoría de estos empleos destruidos pertenecen al segmento de población con menor nivel de formación y estudios. El sector energético, sin embargo, ve incrementar su población ocupada. En este sector destacan Madrid, donde crecen los empleos sobre todo de estudios anteriores al superior y estudios superiores, y Andalucía, donde el aumento de empleos se produce en el segmento de bachiller elemental/EGB/ESO, formación profesional I/ciclos formativos de grado medio y bachiller superior/BUP y COU/ bachillerato, formación profesional II/ciclos formativos de grado superior. El sector manufacturero soporta una intensa destrucción de empleos, en la clasificación de población con menor formación y estudios, destacando Cataluña, Comunidad Valenciana, Andalucía y Madrid. La mayor expulsión de empleos se produce en el sector de la construcción, especialmente en aquella parte de población con menor formación y nivel de estudios. En este caso, destacan Andalucía, Cataluña, Comunidad Valenciana y Madrid. El sector servicios destinados a la venta ve disminuido el empleo principalmente en la población con menor formación; aquí se resaltan las regiones de Cataluña, Andalucía y Canarias. Y el sector servicios no destinado a la venta experimenta un incremento del empleo, esencialmente en la población con mayor nivel de formación, como son Andalucía, Cataluña, Madrid y Comunidad Valenciana.

Los sectores más afectados por la crisis en el periodo analizado han sido, por el lado de la dinámica experimentada por el PIB, el sector industrial y el sector de la construcción, mientras que por el lado de la evolución de los empleos, los sectores que han contribuido con mayor intensidad a la destrucción de puestos de trabajo han sido los dos sectores citados anteriormente y el sector servicios privados (tabla 9). Con respecto al crecimiento del PIB, las regiones con mayor contribución en el deterioro de la generación de riqueza experimentado por el sector industrial han sido Navarra, País Vasco, Galicia y La Rioja, mientras que por el sector de la construcción han destacado Extremadura, Navarra, Baleares y Andalucía.

Tabla 9.

Contribución de cada sector en el crecimiento del PIB y del empleo regional en el periodo 2007-2010 (tasa de variación media anual acumulativa en \%)

\begin{tabular}{|c|c|c|c|c|c|c|c|}
\hline \multicolumn{7}{|c|}{ Contribución sectorial en el crecimiento del PIB } \\
\hline Regiones & $\begin{array}{c}\text { Agricul. } \mathbf{y} \\
\text { pesca }\end{array}$ & $\begin{array}{c}\text { Energía } \mathbf{y} \\
\text { agua }\end{array}$ & Industria & Constr. & Serv. priv. & Serv. púb. & Total \\
\hline Andalucía & $-5,23$ & 3,26 & 63,34 & 77,93 & $-11,30$ & $-33,07$ & 100,00 \\
\hline Aragón & 4,68 & $-0,97$ & 106,28 & 24,12 & $-18,59$ & $-20,57$ & 100,00 \\
\hline Asturias & 1,79 & 16,18 & 91,06 & 41,58 & $-37,36$ & $-18,45$ & 100,00 \\
\hline Baleares & $-0,07$ & 3,76 & 78,00 & 91,99 & $-39,67$ & $-39,69$ & 100,00 \\
\hline Canarias & 5,01 & 0,34 & 21,60 & 53,74 & 47,15 & $-29,91$ & 100,00 \\
\hline Cantabria & 3,34 & $-2,46$ & 118,99 & 67,60 & $-56,87$ & $-35,93$ & 100,00 \\
\hline Castilla y León & 1,58 & 22,47 & 75,58 & 37,46 & $-9,09$ & $-32,47$ & 100,00 \\
\hline Castilla-La Mancha & 0,05 & 0,29 & 99,33 & 69,38 & $-15,31$ & $-60,58$ & 100,00 \\
\hline Cataluña & $-0,72$ & 5,18 & 92,77 & 28,85 & $-15,60$ & $-14,47$ & 100,00 \\
\hline
\end{tabular}


IMPACTO DE LA CRISIS ECONÓMICA EN EL EMPLEO DE LAS REGIONES ESPAÑOLAS: UN ANÁLISIS SECTORIAL EN EL PERIODO 2007-2010

Continuación

\begin{tabular}{|c|c|c|c|c|c|c|c|}
\hline \multicolumn{8}{|c|}{ Contribución sectorial en el crecimiento del PIB } \\
\hline Regiones & $\begin{array}{c}\text { Agricul. y } \\
\text { pesca }\end{array}$ & $\begin{array}{c}\text { Energía y } \\
\text { agua }\end{array}$ & Industria & Constr. & Serv. priv. & Serv. púb. & Total \\
\hline C. Valenciana & $-1,29$ & $-5,61$ & 104,83 & 43,32 & $-21,68$ & $-24,72$ & 100,00 \\
\hline Extremadura & $-7,38$ & 1,27 & 127,11 & 146,47 & $-42,99$ & $-136,66$ & 100,00 \\
\hline Galicia & $-7,99$ & $-2,23$ & 146,14 & 62,29 & $-54,58$ & $-52,34$ & 100,00 \\
\hline Madrid & 0,07 & 2,62 & 102,69 & 69,31 & $-40,48$ & $-40,63$ & 100,00 \\
\hline Murcia & $-13,42$ & 2,42 & 89,82 & 54,52 & $-8,81$ & $-30,14$ & 100,00 \\
\hline Navarra & $-35,08$ & 0,37 & 330,84 & 94,70 & $-173,11$ & $-129,83$ & 100,00 \\
\hline País Vasco & $-6,04$ & $-3,57$ & 190,94 & 49,65 & $-68,67$ & $-68,28$ & 100,00 \\
\hline La Rioja & $-1,74$ & $-0,72$ & 141,68 & 34,89 & $-45,48$ & $-33,52$ & 100,00 \\
\hline España & $-1,54$ & 3,33 & 94,16 & 51,07 & $-20,91$ & $-30,99$ & 100,00 \\
\hline \multicolumn{8}{|c|}{ Contribución sectorial en el crecimiento del empleo } \\
\hline Regiones & $\begin{array}{c}\text { Agricul. y } \\
\text { pesca }\end{array}$ & $\begin{array}{c}\text { Energía y } \\
\text { agua }\end{array}$ & Industria & Constr. & Serv. priv. & Serv. púb. & Total \\
\hline Andalucía & 22,39 & $-3,52$ & 14,38 & 48,07 & 26,03 & $-12,31$ & 100,00 \\
\hline Aragón & 12,55 & $-5,93$ & 39,05 & 35,58 & 34,56 & $-17,07$ & 100,00 \\
\hline Asturias & 17,79 & $-25,62$ & 28,71 & 58,12 & 37,05 & $-19,19$ & 100,00 \\
\hline Baleares & 2,75 & $-3,15$ & 11,76 & 35,69 & 55,98 & $-4,88$ & 100,00 \\
\hline Canarias & 0,72 & $-2,52$ & 7,15 & 38,60 & 53,30 & $-0,35$ & 100,00 \\
\hline Cantabria & 21,64 & $-8,43$ & 25,73 & 56,12 & 20,07 & $-17,88$ & 100,00 \\
\hline Castilla y León & 24,48 & $-14,87$ & 34,19 & 64,21 & 13,62 & $-25,75$ & 100,00 \\
\hline Castilla-La Mancha & 15,58 & $-5,02$ & 27,36 & 55,51 & 19,07 & $-15,54$ & 100,00 \\
\hline Cataluña & 3,04 & $-3,18$ & 41,08 & 34,25 & 32,45 & $-9,87$ & 100,00 \\
\hline C. Valenciana & 3,64 & $-2,11$ & 30,44 & 34,13 & 37,70 & $-6,58$ & 100,00 \\
\hline Extremadura & 47,00 & $-8,74$ & 18,66 & 73,30 & 0,48 & $-33,29$ & 100,00 \\
\hline Galicia & 24,63 & $-9,83$ & 39,14 & 53,22 & 7,21 & $-16,89$ & 100,00 \\
\hline Madrid & $-0,81$ & $-6,45$ & 28,18 & 53,59 & 31,85 & $-9,65$ & 100,00 \\
\hline Murcia & 13,02 & $-2,50$ & 19,22 & 46,64 & 28,74 & $-8,69$ & 100,00 \\
\hline Navarra & 16,12 & $-5,82$ & 49,43 & 50,62 & 4,71 & $-17,16$ & 100,00 \\
\hline País Vasco & 6,24 & $-6,95$ & 62,62 & 33,46 & 25,25 & $-22,38$ & 100,00 \\
\hline La Rioja & 29,52 & $-2,94$ & 43,28 & 30,51 & 14,96 & $-16,31$ & 100,00 \\
\hline España & 10,19 & $-4,68$ & 28,17 & 43,73 & 30,88 & $-11,15$ & 100,00 \\
\hline
\end{tabular}

Fuente: elaboración de los autores. 
Centrando la atención en la reducción del empleo, las regiones que con mayor fuerza han contribuido en el intenso proceso de pérdidas de empleo en el sector constructor han sido, especialmente, Extremadura, Castilla y León, Asturias, Cantabria y Castilla-La Mancha. En el sector servicios privados se han destacado Baleares, Canarias, Comunidad Valenciana y Asturias. Y las regiones que más intensamente han participado en el deterioro del empleo en el sector industrial han sido País Vasco, Navarra, La Rioja, Cataluña, Galicia, Aragón y Castilla y León. Sin embargo, la reducción del PIB se ha visto frenada por los sectores servicios públicos y servicios privados, que han contribuido restando la tendencia decreciente de la generación de riqueza en la economía española. En el proceso de destrucción de empleos, los sectores que han disminuido la enérgica reducción de estos han sido los sectores servicios públicos y energía y agua.

Si se enfoca la contribución del crecimiento del PIB y del empleo a nivel regional, y si se hace referencia a la evolución del PIB, se observa que las comunidades autónomas que han contribuido más intensamente han sido Cataluña, Andalucía, Madrid y Comunidad Valenciana (tabla 10). Respecto al empleo, las regiones con mayor participación en la destrucción de empleo han sido, hasta 2010, Cataluña, Andalucía, Comunidad Valenciana y Madrid. Por tanto, en este aspecto coinciden las regiones que más han contribuido en el deterioro de la economía española, tanto a través del PIB como en el empleo.

No obstante lo anterior, no todas las regiones contribuyen con la misma intensidad ni en todos los sectores. De hecho, Andalucía participa en la reducción de la actividad productiva y del empleo en prácticamente todos los sectores productivos, del mismo modo que Cataluña y Madrid, aunque estas últimas no destacan por su contribución en la agricultura y pesca. Así mismo, la Comunidad Valenciana contribuye especialmente en algunos sectores productivos, como son la industria, la construcción y los servicios privados.

A continuación se presenta un análisis shift-share con el fin de valorar, en la evolución del empleo durante el periodo estudiado, las diferencias existentes a nivel sectorial y las experimentadas a nivel regional. Se trata, en definitiva, de descomponer las diferencias que se han producido en la evolución del empleo en el periodo 2007-2010 en la contribución de los sectores y la contribución territorial a partir de la siguiente expresión:

$$
C N=C E+C D
$$

siendo:

$$
\begin{gathered}
C N=r_{j}-r \\
C E=\sum_{i}\left(s_{i j}-s_{i}\right) * r_{i} \\
C D=\sum_{i}\left(r_{i j}-r_{i}\right)^{*} s_{i j}
\end{gathered}
$$

Donde $\mathrm{CN}$ representa el cambio neto experimentado por el empleo en el periodo analizado; $C E$ constituye el cambio estructural o sectorial ${ }^{8}$; CD, el cambio diferencial"; " $i$ " y " $j$ " denotan, respectivamente, el sector y la región; " $r$ ", el crecimiento de las variables consideradas en el periodo estudiado, y "s", la proporción de empleo de cada sector y región respecto al conjunto de los empleos totales del conjunto de las regiones españolas.

Los hechos estilizados, provenientes de la aplicación de esta técnica, se presentan en la tabla 11.

Los resultados obtenidos indican que existen algunas regiones cuyo empleo ha sufrido un

8 Mide el impacto de la diferencia en el cambio producido en la estructura sectorial del empleo de las distintas regiones españolas, bajo el supuesto de que el cambio producido en el empleo de cada sector es el mismo en las distintas regiones españolas. Tomará valores positivos si las regiones están relativamente especializadas en sectores $\left(s_{i j}-s_{i}\right)$ con elevados niveles de empleos. En efecto, alcanzará su valor máximo si un determinado territorio se encuentra completamente especializado en el sector con mayor empleo por término medio.

9 Recoge la proporción de los cambios regionales experimentados por el empleo en el periodo analizado atribuibles a la evolución del empleo sectorial de las regiones españolas respecto a la media nacional, suponiendo que la estructura del empleo de las regiones españolas fuese igual a la media regional. Tomará valores positivos si la región presenta variaciones sectoriales del empleo superiores a la media nacional $\left(r_{i j}-r_{i}\right)$ 
IMPACTO DE LA CRISIS ECONÓMICA EN EL EMPLEO DE LAS REGIONES ESPAÑOLAS: UN ANÁLISIS SECTORIAL EN EL PERIODO 2007-2010

Tabla 10

Contribución de cada región en el crecimiento del PIB y del empleo sectorial en el periodo 2007-2010

(tasa de variación media anual acumulativa en \%)

\begin{tabular}{|c|c|c|c|c|c|c|c|}
\hline \multicolumn{8}{|c|}{ Contribución regional al crecimiento del PIB } \\
\hline Regiones & $\begin{array}{l}\text { Agricul. y } \\
\text { pesca }\end{array}$ & $\begin{array}{c}\text { Energía y } \\
\text { agua }\end{array}$ & Industria & Constr. & Serv. priv. & Serv. púb. & Total \\
\hline Andalucía & 50,56 & 14,58 & 9,99 & 22,64 & 8,04 & 15,86 & 14,86 \\
\hline Aragón & $-13,91$ & $-1,33$ & 5,15 & 2,16 & 4,06 & 3,03 & 4,57 \\
\hline Asturias & $-3,28$ & 13,68 & 2,72 & 2,29 & 5,03 & 1,67 & 2,81 \\
\hline Baleares & 0,06 & 1,44 & 1,05 & 2,29 & 2,41 & 1,63 & 1,27 \\
\hline Canarias & $-15,41$ & 0,49 & 1,09 & 4,97 & $-10,66$ & 4,56 & 4,73 \\
\hline Cantabria & $-2,26$ & $-0,77$ & 1,31 & 1,38 & 2,83 & 1,21 & 1,04 \\
\hline Castilla y León & $-6,14$ & 40,22 & 4,79 & 4,38 & 2,59 & 6,25 & 5,96 \\
\hline Castilla-La Mancha & $-0,12$ & 0,31 & 3,68 & 4,75 & 2,56 & 6,83 & 3,49 \\
\hline Cataluña & 12,28 & 41,03 & 25,95 & 14,88 & 19,65 & 12,30 & 26,34 \\
\hline C. Valenciana & 8,94 & $-18,03$ & 11,89 & 9,06 & 11,08 & 8,52 & 10,68 \\
\hline Extremadura & 3,02 & 0,24 & 0,85 & 1,80 & 1,29 & 2,77 & 0,63 \\
\hline Galicia & 17,97 & $-2,32$ & 5,36 & 4,22 & 9,02 & 5,84 & 3,46 \\
\hline Madrid & $-0,58$ & 10,15 & 14,05 & 17,49 & 24,96 & 16,90 & 12,89 \\
\hline Murcia & 24,91 & 2,08 & 2,72 & 3,04 & 1,20 & 2,77 & 2,85 \\
\hline Navarra & 11,05 & 0,05 & 1,70 & 0,90 & 4,01 & 2,03 & 0,48 \\
\hline País Vasco & 12,83 & $-3,50$ & 6,63 & 3,18 & 10,72 & 7,19 & 3,26 \\
\hline La Rioja & 0,70 & $-0,14$ & 0,94 & 0,42 & 1,35 & 0,67 & 0,62 \\
\hline España & 100,00 & 100,00 & 100,00 & 100,00 & 100,00 & 100,00 & 100,00 \\
\hline \multicolumn{8}{|c|}{ Contribución regional al crecimiento del empleo } \\
\hline Regiones & $\begin{array}{c}\text { Agricul. y } \\
\text { pesca }\end{array}$ & $\begin{array}{c}\text { Energía y } \\
\text { agua }\end{array}$ & Industria & Constr. & Serv. priv. & Serv. púb. & Total \\
\hline Andalucía & 39,93 & 13,66 & 9,29 & 19,69 & 15,35 & 20,11 & 18,20 \\
\hline Aragón & 3,30 & 3,39 & 3,72 & 2,20 & 3,00 & 4,11 & 2,68 \\
\hline Asturias & 2,29 & 7,25 & 1,34 & 1,77 & 1,58 & 2,26 & 1,31 \\
\hline Baleares & 0,80 & 2,02 & 1,24 & 2,43 & 5,38 & 1,30 & 2,97 \\
\hline Canarias & 0,48 & 3,70 & 1,75 & 5,98 & 11,87 & 0,21 & 6,88 \\
\hline Cantabria & 2,07 & 1,75 & 0,89 & 1,26 & 0,63 & 1,56 & 0,97 \\
\hline Castilla-León & 8,54 & 11,27 & 4,32 & 5,26 & 1,57 & 8,22 & 3,56 \\
\hline Castilla-La Mancha & 6,92 & 4,79 & 4,40 & 5,74 & 2,79 & 6,31 & 4,52 \\
\hline Cataluña & 5,55 & 12,60 & 27,11 & 14,56 & 19,54 & 16,45 & 18,59 \\
\hline C. Valenciana & 5,75 & 7,26 & 17,38 & 12,40 & 19,64 & 9,51 & 16,09 \\
\hline Extremadura & 5,90 & 2,39 & 0,85 & 2,17 & 0,02 & 3,83 & 1,28 \\
\hline Galicia & 8,77 & 7,60 & 5,04 & 4,48 & 0,85 & 5,49 & 3,63 \\
\hline Madrid & $-0,81$ & 14,01 & 10,20 & 12,57 & 10,51 & 8,82 & 10,20 \\
\hline Murcia & 5,31 & 2,24 & 2,84 & 4,35 & 3,87 & 3,24 & 4,16 \\
\hline Navarra & 1,41 & 1,10 & 1,57 & 1,04 & 0,14 & 1,37 & 0,89 \\
\hline País Vasco & 1,92 & 4,63 & 6,96 & 2,43 & 2,56 & 6,27 & 3,13 \\
\hline La Rioja & 1,92 & 0,42 & 1,02 & 0,47 & 0,32 & 0,97 & 0,66 \\
\hline España & 100,00 & 100,00 & 100,00 & 100,00 & 100,00 & 100,00 & 100,00 \\
\hline
\end{tabular}

Fuente: elaboración de los autores. 
Tabla 11

\begin{tabular}{|c|c|c|c|}
\hline \multicolumn{5}{|c}{ Análisis shift-share de la evolución del empleo sectorial (2007-2010) } \\
\hline Región & CN & CE & CD \\
\hline Andalucía & $-1,82524886$ & 0,39764985 & $-0,43322686$ \\
\hline Aragón & $-0,47199191$ & $-0,03876505$ & 1,78389731 \\
\hline Asturias & 2,60499599 & 0,82109868 & 2,06178688 \\
\hline Baleares & 1,95386047 & $-0,10792641$ & $-7,68409006$ \\
\hline Canarias & $-6,28323121$ & 1,40085884 & 1,90223978 \\
\hline Cantabria & 1,57420773 & $-0,32803205$ & 2,14289537 \\
\hline Castilla y León & 2,36160996 & 0,21871459 & 2,52573862 \\
\hline Castilla-La Mancha & 1,49957039 & $-1,02616822$ & $-0,11658652$ \\
\hline Cataluña & $-1,27051544$ & $-1,15392892$ & $-1,89480960$ \\
\hline Comunidad Valenciana & $-3,55380994$ & $-1,65900034$ & 0,32780502 \\
\hline Extremadura & 1,72327910 & 1,39547408 & 1,57317411 \\
\hline Galicia & 1,58877831 & 0,01560420 & 1,36681615 \\
\hline Madrid & 3,54150406 & 2,17468791 & 0,51007440 \\
\hline Murcia & $-1,12053305$ & $-1,63060745$ & 4,62677077 \\
\hline Navarra & 3,14302067 & $-1,48375010$ & 4,08242909 \\
\hline País Vasco & 4,12378614 & 0,04135705 & $-1,382297291083$ \\
\hline
\end{tabular}

Fuente: elaboración de los autores.

descenso superior a la media del conjunto de las regiones españolas, como Canarias $(-6,28)$, Comunidad Valenciana $(-3,55)$, Andalucía $(-1,82)$, Cataluña $(-1,27)$, Murcia $(-1,12)$ y Aragón $(-0,47)$. Ahora bien, no todas ellas presentan la disminución del empleo del mismo modo, pues Canarias, Andalucía, y Aragón presentan un alto componente diferencial, síntoma de que la caída en el empleo ha sido fruto, principalmente, de la intensidad de la disminución regional del empleo, pues se supone que no existen cambios en las estructuras sectoriales del empleo. Sin embargo, Cataluña y Murcia muestran un alto componente estructural dentro del cambio neto, lo que indica que las disminuciones del empleo han sido más intensas en algunos sectores en concreto, como es el caso del sector industrial en Cataluña y del sector de la construcción en Murcia. Se destaca el caso de la
Comunidad Valenciana, pues la disminución del empleo experimentada en el empleo en el periodo analizado es debido al componente estructural y al diferencial, lo que indica que no solo ha disminuido el empleo por encima de la media regional de forma destacada, sino que además ha disminuido en ciertos sectores, como son el industrial y el de la construcción.

Por otro lado, se encuentran las regiones que han reducido el empleo por debajo de la media del conjunto de las regiones españolas, como son País Vasco $(4,12)$, Madrid $(3,54)$, Navarra $(3,14)$, Asturias $(2,60)$, Castilla y León $(2,36)$, Baleares $(1,95)$, Extremadura $(1,73)$, La Rioja $(1,62)$, Galicia $(1,59)$, Cantabria $(1,57)$ y Castilla-La Mancha $(1,50)$; hecho que indica que la crisis les ha afectado con menor intensidad, al menos en la cuestión del empleo. Ahora bien, no todas ellas han contraído el empleo 
de la misma forma, ya que Asturias, Castilla y León, Galicia y País Vasco han presentado un componente diferencial más destacado; Extremadura y Madrid lo han sustentado en el componente estructural y Baleares, Castilla-La Mancha, Navarra y La Rioja, a pesar de tener datos negativos en el componente estructural, han tenido un componente neto positivo debido a su alto valor en el componente diferencial. Lo anterior muestra que su crecimiento menor a la media regional se ha basado fundamentalmente en la dinámica del empleo regional supuesta una estructura sectorial común.

Por lo anterior, se observa que no todas las regiones han experimentado el mismo pulso a la crisis económica, pues existen algunas cuya pérdida de empleo ha sido superior a la media regional española, y otras que han destruido empleo por debajo de dicha media. No obstante, resulta necesario indicar que, independientemente del grupo en el que se encuadren dichas regiones, no todas han soportado la pérdida de empleo del mismo modo, ya que algunas lo han sustentado en una pérdida diferencial y otras, en función de su estructura sectorial.

Teniendo en cuenta que este trabajo trata de analizar el impacto de la crisis económica en los distintos sectores de la economía española, y con el fin de conocer el grado de influencia de la evolución del PIB en la dinámica del empleo, se ha realizado una estimación sectorial en la que se relacionan los

Tabla 12.

\begin{tabular}{|c|c|c|c|c|c|c|}
\hline \multicolumn{7}{|c|}{$\begin{array}{l}\text { Relación entre empleos y PIB años 2007-2010 (modelos de regresión con datos en panel) } \\
\text { Variable dependiente: log (empleos) } \\
\text { MCO }\end{array}$} \\
\hline Sectores productivos & Constante & Coeficiente & $R^{2}$ ajustado & DW & F-Estadístico & $\mathbf{n}$ \\
\hline Agricultura y pesca & $4,307852(* * *)$ & $0,918786(* * *)$ & 0,905458 & 1,863238 & 336,21 & 72 \\
\hline Energía y agua & $2,059881(* * *)$ & $0,990325(* * *)$ & 0,949009 & 1,975821 & 429,06 & 72 \\
\hline Industria transformadora & $3,797756(* * *)$ & $0,937754(* * *)$ & 0,979903 & 2,000227 & $1.707,51$ & 72 \\
\hline Construcción & $3,837788(* * *)$ & $0,925655(* * *)$ & 0,971845 & 1,876583 & $1.209,11$ & 72 \\
\hline Servicios privados & $3,351856\left(^{(* * *}\right)$ & $0,978175(* * *)$ & 0,980755 & 1,805102 & $3.619,29$ & 72 \\
\hline Servicios públicos & $3,710798(* * *)$ & $0,962114(* * *)$ & 0,994574 & 1,951289 & $4.216,71$ & 72 \\
\hline Total sectores & $3,531376(* * *)$ & $0,970567(* * *)$ & 0,983958 & 1,767466 & $4.355,82$ & 72 \\
\hline
\end{tabular}

Nota:

(*) Significativo al $10 \%$

$(* *)$ Significativo al $5 \%$.

$(* * *)$ Significativo al $1 \%$.

Fuente: elaboración de los autores. 
empleos y el PIB de las regiones españolas (tabla $12)^{10}$. A través de estas relaciones se podrá, de cierta manera, establecer la influencia que ha tenido la crisis económica en el nivel de empleo regional de los distintos sectores productivos.

Las regresiones elaboradas a partir de datos en panel relacionan el logaritmo del PIB y el de los empleos, e intentan reflejar las diferencias existentes en cada uno de los sectores productivos. Las estimaciones han sido corregidas de autocorrelación, en los casos en los que ha sido necesario, y de heterocedasticidad mediante el procedimiento de White ${ }^{11}$. Los coeficientes que presentan son fuertemente significativos y los modelos son explicativos.

Los resultados obtenidos a partir de las regresiones planteadas anteriormente indican, por un lado, que la actividad productiva ha influido positivamente en el empleo, como era de esperar; por otro lado, que las diferencias existentes entre los sectores productivos en el periodo analizado son manifiestas. De lo anterior se puede deducir que la evolución del PIB no ha tenido el mismo impacto en el empleo en todos los sectores productivos, por lo que podría considerarse como un elemento que está afectando a las diferencias existentes en la actividad económica y, por ende, en los desequilibrios producidos en el nivel de desarrollo económico sectorial y regional en España. En cierto sentido, según los resultados que arrojan las estimaciones realizadas, la evolución del PIB ( $y$, por tanto, uno de los indicadores ampliamente

10 Dicha relación se ha establecido a partir de la siguiente expresión:

$\log \left(E_{i}\right)=\beta_{1}+\beta_{2} \log \left(Y_{i}\right)+u_{i}$

siendo $E$ el empleo y $Y$ el Producto Interno Bruto de cada una de las regiones españolas, y $u$ los errores de los datos de la regresión. En dicho contraste se han incluido no sólo los valores regionales, sino también los valores totales de la economía española.

11 La aplicación del contraste de endogeneidad de las variables explicativas mediante el test de Granger y el test de Hausman evidencia la existencia de problemas entre las variables consideradas. Ante esta situación se decide abordar el método de momentos generalizados (GMM). La variable instrumental utilizada ha sido la calificada como potencialmente endó gena por dicho test retardado un periodo (Blundell y Bond 2000; Blundell et al., 2000). Las diferencias de los resultados obtenidos no han variado sustancialmente con respecto a los presentados en la tabla 12 .

Finanz. polit. econ., ISSN 2248-6046, Vol. 6, No. 1, enero - junio, 2014, pp. 43 - 71 Edición Especial - Economía Regional utilizado para valorar la situación económica) ha tenido un mayor impacto en el empleo de los sectores energía y agua, servicios privados y servicios públicos. Lo cierto es que esta última premisa abre una interesante línea de investigación para posteriores trabajos científicos que indaguen en la participación y el impacto que la actividad productiva a nivel sectorial podría tener en la reducción de las disparidades económicas en las comunidades autónomas y, por tanto, en la cohesión económica y social de las regiones españolas.

\section{CONCLUSIONES}

El objetivo del presente trabajo ha sido analizar el impacto de la crisis económica en las distintas regiones y en los distintos sectores en las comunidades autónomas españolas, a partir del empleo y el capital humano, para poder establecer una clasificación de las regiones según la repercusión que sobre éstas haya podido tener la crisis económico-financiera. Las ideas y reflexiones más relevantes que se desprenden del estudio realizado se exponen a continuación.

1. La crisis económico-financiera comienza a repercutir claramente en la economía española a partir del segundo trimestre de 2008, cuando el PIB, el empleo y el número de horas trabajadas comienzan a disminuir de forma relevante.

2. La elasticidad del empleo a las variaciones del nivel de desarrollo económico (medido a partir del PIBpc) es cuantitativamente más significativo en la economía española que en la media de la UE-27. En efecto, la relación entre la reducción del PIBpc en el periodo estudiado 2007-2010 y el incremento de la tasa de desempleo en la UE-27 fue de 2,5 , mientras que este mismo indicador en la economía española alcanzó la cifra de 6, más del doble de la media europea.

3. El impacto de la crisis económica ha sido desigual en las distintas regiones españolas, lo que pone de cuestión la intención y 
el esfuerzo realizado en las décadas anteriores para lograr la cohesión económica y social de los territorios de la economía española. Las regiones que han acusado con mayor intensidad los efectos de la crisis económica en términos de PIBpc, número de horas trabajadas y tasa de desempleo han sido Andalucía, Aragón, Baleares, Canarias, Castilla-La Mancha, Comunidad Valenciana y Murcia; además, han sido las regiones más castigadas por el descenso de la actividad económica, salvo Aragón, ya que en el periodo considerado, por lo general, presentaban un nivel de desarrollo económico inferior a la media de las regiones españolas.

4. Cataluña y Madrid, han experimentado en el periodo analizado un incremento del número de empresas sin asalariados (que supone un $55 \%$ del total de empresas creadas), y La Rioja y Castilla y León (con una creación de cuatro empresas con más de 500 empleados cada una), se han destacado como regiones con un importante espíritu emprendedor. Por el contrario, Comunidad Valenciana, Andalucía, Murcia y Canarias sobresalen por la pérdida de pequeñas, medianas y grandes empresas.

5. La pérdida de empleos ha sido generalizada en todas las regiones españolas, tanto en empleos asalariados como en los no asalariados. Las regiones que han sufrido mayores pérdidas de empleo, sobre todo de empleos asalariados, han sido Canarias, Comunidad Valenciana, Murcia, Andalucía, Baleares, Cataluña y Castilla-La Mancha.

6. La crisis económica ha afectado, de forma general, a todos los segmentos de población según el nivel de estudios, pero el grado de incidencia ha sido destacadamente mayor en los trabajadores cuanto menor ha sido su nivel de formación. Incluso se podría decir que los sectores en los que se produce un crecimiento del empleo en el periodo 2007-2010, este se pone de manifiesto principalmente en aquella parte de población con mayor nivel de formación.

7. La economía española ha perdido $1,8 \mathrm{mi}-$ llones de empleos entre 2007 y 2010 , de los cuales el $46 \%$ corresponde a empleos del sector de la construcción, presumiblemente el más afectado por la crisis. El sector servicios privados redujo el empleo un $30,6 \%$, mientras que el sector industrial lo vio disminuir el $28,3 \%$. Por tanto, la crisis económica ha afectado especialmente a un sector que históricamente ha tenido un mayor nivel de productividad del empleo, lo que sin duda debilita y pone en entredicho el modelo productivo español.

8. Por sectores productivos, la agricultura y pesca ha sufrido una fuerte reducción del empleo en Andalucía, Galicia, Castilla y León y Castilla-La Mancha. El sector industrial ha disminuido el empleo en Comunidad Valenciana, Madrid, Andalucía y País Vasco. La pérdida de empleos del sector de la construcción se ha hecho sentir sobre todo en Andalucía, Cataluña, Comunidad Valenciana y Madrid. $\mathrm{Y}$ las regiones más afectadas por la crisis en el sector servicios privados han sido Comunidad Valenciana, Cataluña, Andalucía, Canarias y Madrid.

9. La evolución del PIB (uno de los indicadores más utilizados para valorar la crisis económica) ha influido - como predice la teoría económica - en el nivel de empleo. Esta relación se ha dado con mayor intensidad en los empleos de los sectores energía y agua, servicios privados y servicios públicos. Estas diferencias sectoriales podrían estar afectando, sin duda, a las disparidades económicas existentes entre las comunidades autónomas españolas.

Teniendo en cuenta las conclusiones expuestas anteriormente, se hace necesario que las 
administraciones, tanto central como autonómicas, realicen un serio esfuerzo para conseguir frenar los efectos de la crisis económica sobre el empleo. En este sentido, sería interesante planificar y ejecutar políticas activas de empleo que, por un lado, frenen la sangría del desempleo y que, por otro lado, permitan mediante la adaptación y formación de la población activa a los nuevos cambios tecnológicos e innovadores la reinserción en el mercado laboral de la población desempleada, para posibilitar así una mayor resistencia al desempleo. Así mismo, se estima oportuna la aplicación de políticas económicas nacionales y autonómicas que permitan reactivar la demanda, así como el establecimiento de un horizonte político, social y económico claro que estimule la inversión generadora de empleo en una economía que presenta, en la actualidad, una fuerte tasa de desempleo.
Por último, a pesar de lo anterior, se estima que la investigación no ha concluido aún, pues quedan aspectos que no han sido tratados, o en los que es necesario profundizar, a través de estudios más exhaustivos o de análisis de casos, región por región y sector por sector, que pongan de relieve ciertas cuestiones que quedan ocultas en los análisis más generales y agregados, y otras que solo quedan al descubierto cuando la investigación desciende a niveles de mayor desagregación. Se confía en que la ampliación del periodo muestral, la utilización de nuevas fuentes estadísticas, distintos métodos de investigación y, sobre todo, la elaboración de variables que aproximen mejor los conceptos teóricos permitan en un futuro próximo corroborar la validez de estas conclusiones.

\section{REFERENCIAS}

1. Alcaide, P. (2011). Balance Económico Regional (Autonomías y Provincias). Años 2000 a 2010. Madrid: Fundación de las Cajas de Ahorros.

2. Blundell, R. y Bond, S. (2000). GMM Estimation with persistent panel data: an application to production functions. Econometric Reviews, 19(3), 321-340.

3. Blundell, R., Bond, S. y Windmeijer, F. (2000). Estimation in dynamic panel data models: improving on the performance of the standard GMM estimator. Institute for Fiscal Studies, Working Paper W00/12.

4. Boldrín, M., Conde-Ruiz, I. y Díaz-Giménez, J. (2010): Eppur si muove! España: creciendo sin un modelo. En S. Bentolila, M. Boldrín, J. Díaz-Giménez y J.J. Dolado, (Eds.), La crisis de la economía española. Análisis económico de la gran recesión (pp. 165-246). Madrid: Fedea.

5. Boura, S. (2013). Who are the victims of the crisis? The social impact of the crisis on youth unemployed university graduates from Greece and Spain in a European comparative analysis. SSRN Working Paper Series, Rochester. Recuperado de http://dx.doi.org/10.2139/ssrn.2210366

6. Cabrales, A., Dolado, J.J., Felgueroso, F. y Vázquez, P. (2009). La crisis de la economía española: lecciones y propuestas. Madrid: Fedea.

7. Cuadrado, J.R. y Maroto, A. (2012). El problema de la productividad en España: causas estructurales, cíclicas y sectoriales. Madrid: Fundación de las Cajas de Ahorros.

8. Dabán, T., Díaz, A., Escribá, J. y Murgui, M.J. (2002). La base de datos BD.MORES. Revista de Economía Aplicada, X(30), 165-184.

9. Díaz, A. y García, E. (2011). Notas metodológicas de la Base de Datos Macroeconómicos de España (Bdmacro). Secretaría de Estado de Presupuestos y Gastos del Ministerio de Hacienda y Administraciones Públicas del Gobierno de España. Recuperado de http://www.sepg.pap.minhap. gob.es/sitios/sepg/es-ES/Presupuestos/Documentacion/-paginas/bdmacro.aspx. 
10. Doménech, R. y García, J.R. (2010). ¿Cómo conseguir que crezcan la productividad y el empleo, y disminuya el desequilibrio exterior? WP Economic Research Department. Recuperado de http://www.bbvaresearch.com/KETD/fbin/mult/WP_1007_tcm346-220907.pdf?ts=852010

11. Fernández, R. y Morán, E. (2008). Reacciones fiscales de las Comunidades Autónomas ante una crisis global. Revista Asturiana de Economía, 42, 57-80.

12. Fondo Monetario Internacional (2010). La dinámica del desempleo durante las recesiones y las recuperaciones: La Ley de Okun como punto de partida. En Perspectivas de la economía mundial. Reequilibrar el crecimiento (pp. 75-116). Washington, D.C.: Estudios Económicos Financieros.

13. Fundación Bancaja e Ivie (Instituto Valenciano de Investigaciones Económicas) (2010). Capital humano en España y su distribución provincial. Recuperado de http://www.ivie.es/es/ banco/caphum/series.php

14. Laparra, P. y Pérez, B. (Coords.) (2012). Crisis y fractura social en Europa. Causas y efectos en España. Barcelona: Obra Social "La Caixa".

15. Llusá, R., Feliu, J. y Paunero, X. (Eds.) (2013). Crisis económica e impactos territoriales. Libro de Actas de las V Jornadas de Geografía Económica. Universidad de Girona, Girona.

16. Medina, E., Herrarte, A. y Vicéns, J. (2010). Inmigración y desempleo en España: impacto de la crisis económica. Boletín Económico del ICE, 854, 37-48.

17. Myro, R. (2011). Crisis económica y modelo productivo. Boletín Económico del ICE, 863, $79-94$.

18. Ortega, E. y Peñalosa, J. (2012). Claves de la crisis económica española y retos para crecer en la UEM. Documentos Ocasionales. Recuperado de http://www.bde.es/f/webbde/SES/Secciones/Publicaciones/PublicacionesSeriadas/DocumentosOcasionales/12/Fich/do1201.pdf

19. Peña, A.R. (2007). Análisis sectorial de la productividad y de la estructura productiva en Andalucía. Estudios de Economía Aplicada, 25, 691-726.

20. Peña, A.R. (2008a). Las disparidades económicas regionales en España: las infraestructuras como factor de convergencia en el periodo 1980-2000. Revista de Estudios Regionales, 82, $105-132$

21. Peña, A.R. (2008b). Las disparidades económicas territoriales en España: contribución de los factores productivos al crecimiento regional, 1980-2004. Revista de Información Comercial Española, 844, 205-218.

22. Peña, A.R. (2011). Desarrollo económico regional en España: análisis de la productividad y del empleo como factores determinantes. Boletín Económico del ICE, 3007, 39-48.

23. Peña, A.R. y Jiménez, M. (2012). Convergencia regional en España, 1980-2003: Análisis de la influencia de la eficiencia sectorial y la estructura productiva. Revista Apuntes, 53, 25-50.

24. Peña, A.R. y Jiménez, M. (2013). Productividad y estructura sectorial: elementos determinantes de las disparidades económicas regionales en España. Revista de Estudios Regionales, 97, 137-169.

25. Ruiz-Huerta, J., Vizán, C. y Benyakhlef, M. (2012). Crisis económica y tensión fiscal en las Comunidades Autónomas. En Informe Comunidades Autónomas 2011 (pp. 51-74). Barcelona: Instituto de Derecho Público.

26. Urtasun, A., Izquierdo, M. y Ortega, E. (2012). Un análisis sectorial de la relación entre la actividad y el empleo de la economía española. Boletín Económico, Banco de España.

27. Xiaoni, L., Roca, P. S. y Papaoikonomou, E. (2011). SME's response to the financial and economic crisis and policy implications: an analysis of agricultural and furniture sectors in Catalonia, Spain. Policy Studies, 32, 4, 397-412. 